\title{
Regularity of Harmonic Maps with Prescribed Singularities
}

\author{
Yanyan $\mathrm{Li}^{1 \star}$ and Gang Tian ${ }^{2 \star \star}$ \\ ${ }^{1}$ Department of Mathematics, Princeton University, Princeton, NJ 08544 and Institute for \\ Advanced Study, Princeton, NJ 08540, USA \\ 2 Department of Mathematics, Princeton University, Princeton, NJ 08544, USA
}

Received November 14, 1990, in revised form March 6, 1992

\begin{abstract}
In this paper, we studied the regularity problem for harmonic maps into hyperbolic spaces with prescribed singularities along codimension two submanifolds. This is motivated from one of Hawking's conjectures on the uniqueness of Kerr solutions among all axially symmetric asymptotically flat stationary solutions to the vacuum Einstein equation in general relativity.
\end{abstract}

\section{Introduction}

In the last three decades, much progress has been made on harmonic maps between Riemannian manifolds. Among the outstanding ones, for instance, are the existence of Eells and Sampson [ES] on harmonic maps into nonpositively curved manifolds, with the generalization of R. Hamilton [Ha] to manifolds with boundary, the ones of Sacks and Uhlenbeck [SU], Lemaire [Le] and R. Schoen and S.-T. Yau on harmonic maps defined on Riemann surfaces, and regularity theories of R. Schoen and K. Uhlenbeck [SU1, SU2]. Prior to [SU1, SU2] there had been some regularity theorems due to Hildebrandt, Giusti, Giaquinta (see for example [Gi]) under various assumptions on the target manifolds. These results have brought tremendous new understandings of the geometry of manifolds.

In this paper, we consider the following problem. Let $\left(M, d s^{2}\right)$ be a $n$-dimensional complete Riemannian manifold with or without boundary, and $N \subset M$ be a codimension two closed submanifold; let $h$ be a smooth map from $M \backslash N$ into the naturally compactified hyperbolic space $\bar{H}^{m}$ such that $h(M \backslash N) \subset H^{m}$, where $H^{m}$ is upper-half-space model of $m$-hyperbolic space form. Then we would like to find a harmonic map from $M \backslash N$ in $H^{m}$ with "similar" asymptotic behavior to $h$ along $N$. One natural approach is to perturb $h$ to obtain the harmonic one. To make it

\footnotetext{
* Current address: Department of Mathematics, Rutgers University, New Brunswick, NJ 08903. Research partially supported by a NSF grant DMS-8907849.

$\star \star$ Current address: Courant Institute, 251 Mercer Street, New York, NY 10012. Research partially supported by a NSF grant
} 
precise, we let $\left(y_{1}, \ldots, y_{m}\right)\left(y_{m}>0\right)$ be the global coordinates of $H^{m}$ inherited from $R^{m}$, and $\partial H^{m}=\bar{H}^{m} \backslash H^{m}$ is just defined by $y_{m}=0$. Write $h=\left(h_{1}, \ldots, h_{m}\right)$, then $h_{m} \geqq 0$ and $h_{m}>0$ in $M \backslash N$.

We denote by $H_{1,0}(M)$ (respectively $H_{1, h_{m}, 0}(M)$ ) the completion of $C_{0}^{\infty}(M)$ (resp. $\left.C_{0}^{\infty}(M \backslash N)\right)$ under the norm

$$
\begin{gathered}
\|\psi\|_{H_{1}}^{2}=\int_{M}|\nabla \psi|^{2} d V, \\
\text { (resp. } \left.\|\psi\|_{H_{1}, h_{m}}^{2}=\int_{M} h_{m}^{-2}|\nabla \psi|^{2} d V\right),
\end{gathered}
$$

where $d V$ is the volume form on $M$, and the norm on $\nabla \psi$ is taken with respect to the metric $d s^{2}$ on $M$.

We want to find a harmonic map into $H^{m}$ of form $\left(\varphi_{1}, \ldots, \varphi_{m-1}, h_{m} e^{\varphi_{m}}\right)$ satisfying: $\varphi_{i}-h_{i} \in H_{1, h_{m}, 0}(M)$ for $i=1,2, \ldots, m-1$ and $\varphi_{m} \in H_{1,0}(M)$. Equivalently, we are bound to find the critical point of the functional on $\left(\prod_{i=1}^{m-1}\left(h_{i}+H_{1, h_{m}, 0}(M)\right)\right) \times H_{1,0}(M)$ defined as follows:

$$
\begin{aligned}
F\left(\varphi_{1}, \ldots, \varphi_{m-1}, \varphi_{m}\right)=\int_{M} & {\left[\left|\nabla \varphi_{m}\right|^{2}+\frac{2 \nabla h_{m} \nabla \varphi_{m}}{h_{m}}\right.} \\
& \left.+\left(\sum_{i=1}^{m-1} \frac{\left|\nabla \varphi_{i}\right|^{2}}{h_{m}^{2}} e^{-2 \varphi_{m}}\right)\right] d V .
\end{aligned}
$$

In the case $\log h_{m}$ is harmonic, i.e., $\Delta \log h_{m}=0$ on $M \backslash N$, the second term in the above integration may be taken away from $F$. Under suitable conditions on $h_{1}, \ldots, h_{m}$, one can prove the existence of the minimizer by a standard method (see Sect. 2 or [We1]). For instance, in order to make the functional $F$ meaningful, we have to assume that all integrals $\int_{M} h_{m}^{-2}\left|\nabla h_{i}\right|^{2} d V$ are finite, where $i=1$, $2, \ldots m-1$. In fact, the solution is unique among the admissible functions. See [We1] for more details. One is then led to the problem how regular the critical point $\left(\varphi_{1}, \ldots, \varphi_{m}\right)$ could be along the submanifold $N$ in $M$. This is our main concern in this paper. We demonstrate the smoothness of $\left(\varphi_{1}, \ldots, \varphi_{m}\right)$ under mild nondegeneracy conditions on $h_{m}$. Namely, we show

Theorem 1.1. Let $(M, g)$ be a smooth $n$-dimensional Riemannian manifold without boundary, $N \subset M$ be a smooth $(n-2)$-dimensional closed submanifold, $h_{1}, \ldots$, $h_{m-1}$ be smooth functions on $M, h_{m}>0$ be smooth in $M \backslash N$ and

$$
\begin{gathered}
\Delta \log h_{m}(x)=0, \quad x \in M \backslash N, \\
\lim _{\rho(x) \rightarrow 0} \frac{\log h_{m}(x)}{\alpha \log \rho(x)}=1,
\end{gathered}
$$

where $\rho(x)=\operatorname{dist}(x, N)$ is the distance between $x \in M$ and $N, \alpha>0$ is some positive constant.

Let $\left(\varphi_{1}, \ldots, \varphi_{m}\right)$ be the minimizer of $F$ defined in (1.3) in the space $\left(\prod_{i=1}^{m-1}\left(h_{i}+H_{1, h_{m}, 0}(M)\right)\right) \times\left(H_{1,0}(M) \cap L^{\infty}(M)\right)$. Then for any $\varepsilon>0, \varepsilon<2 \alpha$, $\left(\varphi_{1}, \ldots, \varphi_{m}\right) \in C^{k_{\alpha}, \lambda_{\alpha}}$ with $k_{\alpha}=[2 \alpha-2 \varepsilon]$ and $\lambda_{\alpha}=\min \left\{2 \alpha-2 \varepsilon-k_{\alpha}, 1-\varepsilon\right\}$.

The conclusion of the above theorem still holds if, instead of (1.4), we assume that $\Delta \log h_{m}(x)$ can be extended to $N$ as a smooth function. More generally, if 
$N$ is a submainfold with boundary, this theorem implies that the minimizer $\left(\varphi_{1}, \ldots, \varphi_{m}\right)$ is Hölder continuous in the interior of $N$. But our method also yields Hölder continuity of the minimizer on the boundary of $N$. This is discussed in [LT1].

The existence of such $h_{m}$ is elementary and has been explicitly written down in terms of the Green's function. At those points where $h_{m}>0$, the regularity of $\left(\varphi_{1}, \ldots, \varphi_{m}\right)$ is just the same as that of the harmonic map $\left(h_{1}+\varphi_{1}, \ldots\right.$, $\left.h_{m-1}+\varphi_{m-1}, h_{m} e^{\varphi_{m}}\right)$ into $H^{m}$, and it then follows from the well-known regularity theorem for harmonic maps (cf. [SU1]). Therefore, in order to prove our main theorem, we only need to give the regularity of $\left(\varphi_{1}, \ldots, \varphi_{m}\right)$ at those points where $h_{m}=0$. Some easy computations show that the Euler-Lagrange equation of (1.3) is degenerate at these points. This is the essential difficulty to the proof of Theorem 1.1. The proof we have here is in the spirit of [SU1].

For harmonic maps, it is well known that $C^{\alpha}$-regularity $(\alpha>0)$ automatically implies higher order ones (cf. [Sc]). However it is not clear in our case because of the degeneracy of the Euler-Lagrange equations. Therefore, we also need to derive the regularity estimates of higher order for $\left(\varphi_{1}, \ldots, \varphi_{m}\right)$ from $C^{\alpha}$-estimate $(\alpha>0)$.

We also give an existence theorem of such a harmonic map in case $h_{i}$ $(i=1, \ldots, m-1)$ are constants along the connected components of $N$.

One of our motivations towards such a problem is from one conjecture of Hawking in the formulation of G. Weinstein [We1]. Hawking's conjecture asserts that Kerr solutions are the only asymptotically flat, axially symmetric, stationary ones of Einstein Vacuum Equation in general relativity with certain nondegenerate conditions on event horizons. In case the event horizon is connected, it was settled down by Robinson [Ro] in the 70's. Robinson's proof is based on the uniqueness of harmonic maps into hyperbolic space and earlier work by Ernst and Carter ([Er, $\mathrm{Ca}])$. But this conjecture is still open in general. As an application of our theorem, we will prove that there are no asymptotically flat, axially symmetric, stationary solutions of $E V E$ with disconnected event horizons of small angular momentum.

The organization of this paper is as follows. In Sect. 2, we prove the existence and boundedness of the minimizer of the functional $F$ in (1.3). In Sect. 3, we discuss some total energy estimate. Section 4 contains a modified monotonicity formula for our solutions. Then the Hölder regularity follows from the standard De Giorgi estimate. In Sect. 5, we discuss the regularity of higher order. The application of our theorem is given in the last section (Sect. 6).

Finally, we would like to remark that two possible generalizations can be made in the future. The simpler one is to remove the smoothness condition of $N$; instead we assume that $N$ is a union of submanifolds intersecting with each other transversally. The more interesting one is to lift the assumption on the hyperbolicity of $H^{m}$. In general, when the target may not have nonpositive curvature, one expects the regularity of the minimizer of (1.3) outside a subset of $M$ of codimension 3 as R. Schoen and K. Uhlenbeck found for harmonic maps. All these generalizations will be discussed in the future.

After we finished this work, we learned from G. Weinstein that in the special case where $M=R^{3}, N$ is the $z$-axis, $m=2$ and $\left(\varphi_{1}, \varphi_{2}\right)$ is the minimizer among axisymmetric functions; our theorem is also proved independently by $\mathrm{G}$. Weinstein ([We2]). His method is completely different from ours and seems to be unlikely to be generalized to higher dimensional or nonaxially symmetric cases. 
We would like to thank F.H. Lin for bringing [HL] to our attention. We would also like to thank D. Christodoulou, F.H. Lin and W. Shu for useful discussions on the problem.

\section{Existence}

The existence results in this section are essentially due to G. Weinstein [We1]. We present them here for the sake of completeness.

Let $\left(M^{n}, g\right)$ be a $n$-dimensional $(n \geqq 3)$ smooth compact Riemannian manifold with smooth boundary, $N^{n-2} \subset M^{n}$ be a $(n-2)$-dimensional smooth closed submanifold. Let $\alpha>0$ be some positive number, $\rho(x)$ be a function defined on $M$, $\rho(x)=\operatorname{dist}(x, N)$ for $x$ near $N$ and smooth, strictly positive elsewhere. Let $u$ be the solution of

$$
\begin{aligned}
-\Delta u(x) & =\Delta(\alpha \log \rho(x)), \quad x \in M, \\
\left.u\right|_{\partial M} & =0 .
\end{aligned}
$$

Here $\Delta$ denotes the Laplace-Beltrami operator with respect to the metric of $M$.

It is obvious that $u$ is smooth away from $N$. The question is how smooth it is across $N$. After some essentially elementary calculations, we see that $w$ is at least Hölder continuous. In fact, for any $\varepsilon \in(0,1)$, there exists some positive constant $C(\varepsilon)>0$, such that,

$$
|\nabla u(x)| \leqq C(\varepsilon) \rho(x)^{\varepsilon-1} \quad \forall x \in M \backslash N .
$$

Let $h_{m}(x)=\rho(x)^{\alpha} e^{u(x)}, x \in M$, clearly $\Delta\left(\log h_{m}\right)=0$ on $M \backslash N$.

Let $f_{i}: M \rightarrow \mathbb{R}(1 \leqq i \leqq m)$ be $H^{1}(M)$ function and bounded on $\partial M$. For $i=1,2, \ldots, m-1, f_{i}=0$ on $N$. We look for harmonic maps

$$
\left(\varphi_{1}, \ldots, \varphi_{m-1}, h_{m} e^{\varphi_{m}}\right): M \backslash N \rightarrow H^{m}
$$

with prescribed boundary data:

$$
\begin{aligned}
\left.\varphi_{i}\right|_{\partial M}=f_{i}, & 1 \leqq i \leqq m, \\
\left.\varphi_{i}\right|_{N}=f_{i}=0, & 1 \leqq i \leqq m-1,
\end{aligned}
$$

and the prescribed singularity on $N$ in the sense that $\left|\varphi_{m}\right| \leqq$ constant.

Let us set up the problem rigorously, consider

$$
H\left(\varphi_{1}, \ldots, \varphi_{m-1}, \varphi_{m}\right)=\int_{M}\left\{\left|\nabla \varphi_{m}\right|^{2}+h_{m}^{-2} e^{-2 \varphi_{m}} \sum_{i=1}^{m-1}\left|\nabla \varphi_{i}\right|^{2}\right\} d V,
$$

where

$$
\begin{aligned}
\varphi_{i}-f_{i} & \in H_{1, h_{m}, 0}(M) \text { for } i=1,2, \ldots, m-1, \\
\varphi_{m}-f_{m} & \in H_{0}^{1}(M) .
\end{aligned}
$$

It has been explained that if we can obtain a minimizer of $H\left(\varphi_{1}, \ldots, \varphi_{m}\right)$ among the admissible functions with $\left|\varphi_{m}\right| \leqq$ constant, we will have the harmonic map with prescribed singularity. 
Theorem 2.1. The minimum of $H\left(\varphi_{1}, \ldots, \varphi_{m}\right)$ can be attained among those admissible functions $\varphi_{1}, \ldots, \varphi_{m}$ which satisfy (2.2) and (2.3). Furthermore the minimizer satisfies $\left|\varphi_{m}\right| \leqq$ constant.

Remark 2.1. The condition on the boundary data $f_{i}=0$ on $N(i=1,2, \ldots, m-1)$ can be replaced by $f_{i}=c_{i}$ on $N(i=1,2, \ldots, m-1)$ with $c_{i} \in \mathbb{R}$ being constants. One only needs to make a translation in $\left(\varphi_{1}, \ldots, \varphi_{m-1}\right)$ to achieve this.

Remark 2.2. The assumption of $N^{n-2} \subset M^{n}$ being a closed submanifold can be relaxed to $N^{n-2} \subset M^{n}$ being a submanifold with $\partial N \subset \partial M$, then we need the boundary conditions $\left\{f_{i}\right\}_{i=1}^{m-1}$ to be compatible on $\partial N$ and on $\partial M$. The proof is exactly the same.

Sketch of the Proof of Theorem 2.1. Let $\varphi^{(k)}=\left(\varphi_{1}^{(k)}, \ldots, \varphi_{m-1}^{(k)}, \varphi_{m}^{(k)}\right)$ be a minimizing sequence, with the boundary conditions (2.2) and (2.3). Since $f_{m}$ is bounded on $\partial M$, we can replace $\varphi_{m}^{(k)}$ by $\tilde{\varphi}_{m}^{(k)}=\max \left\{\varphi_{m},-C\right\}$. The new sequence $\varphi^{(k)}=\left(\varphi_{1}^{(k)}, \ldots, \varphi_{m-1}^{(k)}, \tilde{\varphi}_{m}^{(k)}\right)$ will have energy no more than $\varphi^{(k)}$, and the same boundary value as $\varphi^{(k)}$. Therefore $\tilde{\varphi}^{(k)}$ is also a minimizing sequence. We replace $\varphi^{(k)}$ by $\tilde{\varphi}^{(k)}$, but for simplicity, we still denote it as $\varphi^{(k)}$. After this truncation, the minimizing sequence $\varphi^{(k)}$ satisfies

$$
\varphi_{m}^{(k)} \geqq-C
$$

Once we have (2.4), we can use a simple density argument to replace $\varphi_{i}^{(k)}(i=1,2, \ldots, m-1)$ by $\tilde{\varphi}_{i}^{(k)}(i=1,2, \ldots, m-1)$ with $\tilde{\varphi}_{i}^{(k)}-f_{i} \in C_{0}^{\infty}(M \backslash N)$. Once again we replace $\varphi_{i}^{(k)}$ by $\tilde{\varphi}_{i}^{(k)}$, but still keep the old notation.

In order to get an upper bound on $\varphi_{m}^{(k)}$, we explore the isometry group of $H^{m}$. Let us denote

$$
\Phi=\left(\Phi_{1}, \ldots, \Phi_{m-1}, \Phi_{m}\right)=\left(\varphi_{1}, \ldots, \varphi_{m-1}, h_{m} e^{\varphi_{m}}\right) \in H^{m}
$$

We know that

$$
\left\{\begin{array}{l}
\bar{\Phi}_{i}=-\frac{\Phi_{i}}{\Phi_{m}^{2}+\sum_{i=1}^{m-1} \Phi_{i}^{2}} \quad 1 \leqq i \leqq m-1 \\
\bar{\Phi}_{m}=\frac{\Phi_{m}}{\Phi_{m}^{2}+\sum_{i=1}^{m-1} \Phi_{i}^{2}}
\end{array}\right.
$$

is an isometry of $H^{m}$, namely,

$$
\frac{\sum_{i=1}^{m-1} d \Phi_{i}^{2}+d \Phi_{m}^{2}}{\Phi_{m}^{2}}=\frac{\sum_{i=1}^{m-1} d \bar{\Phi}_{i}^{2}+d \bar{\Phi}_{m}^{2}}{\bar{\Phi}_{m}^{2}}
$$

Let us write $\bar{\Phi}$ as

$$
\bar{\Phi}=\left(\bar{\Phi}_{1}, \ldots, \bar{\Phi}_{m-1}, \bar{\Phi}_{m}\right)=\left(\bar{\varphi}_{1}, \ldots, \bar{\varphi}_{m-1}, h_{m}^{-1} e^{\bar{\varphi}_{m}}\right)
$$


It follows easily from (2.7) that

$$
\begin{aligned}
\left|\nabla \varphi_{m}\right|^{2}+\sum_{i=1}^{m-1}\left|\nabla \varphi_{i}\right|^{2} e^{-2 \varphi_{m}} h_{m}^{-2}= & \left|\nabla \bar{\varphi}_{m}\right|^{2}+\sum_{i=1}^{m-1}\left|\nabla \bar{\varphi}_{i}\right|^{2} e^{-2 \bar{\varphi}_{m}} h_{m}^{2} \\
& -2 \nabla\left(\log h_{m}\right) \nabla\left(\varphi_{m}+\bar{\varphi}_{m}\right) .
\end{aligned}
$$

Integrating the above over $M$,

$$
\begin{aligned}
\int_{M}\left|\nabla \varphi_{m}\right|^{2}+\sum_{i=1}^{m-1}\left|\nabla \varphi_{i}\right|^{2} e^{-2 \varphi_{m}} h_{m}^{-2}= & \int_{M}\left|\nabla \bar{\varphi}_{m}\right|^{2}+\sum_{i=1}^{m-1}\left|\nabla \bar{\varphi}_{i}\right|^{2} e^{-2 \bar{\varphi}_{m}} h_{m}^{2} \\
& -2 \int_{M} \nabla\left(\log h_{m}\right) \nabla\left(\varphi_{m}+\bar{\varphi}_{m}\right) .
\end{aligned}
$$

Since $\varphi_{i}^{(k)}=0^{*}(i=1,2, \ldots, m-1)$ near $N$, it follows from (2.6) that $\varphi_{m}+\bar{\varphi}_{m}=0$ near $N$. Using Stokes theorem and the harmonicity of $\log h_{m}$, we have

$$
-2 \int_{M} \nabla\left(\log h_{m}\right) \nabla\left(\varphi_{m}+\bar{\varphi}_{m}\right)=2 \int_{\partial M}\left(\varphi_{m}+\bar{\varphi}_{m}\right) \frac{\partial}{\partial v}\left(\log h_{m}\right)
$$

which depends only on the boundary data $\left\{f_{i}\right\}$. Hence

$$
\begin{gathered}
\int_{M}\left|\nabla \varphi_{m}\right|^{2}+\sum_{i=1}^{m-1}\left|\nabla \varphi_{i}\right|^{2} e^{-2 \varphi_{m}} h_{m}^{-2}= \\
\int_{M}\left|\nabla \bar{\varphi}_{m}\right|^{2}+\sum_{i=1}^{m-1}\left|\nabla \bar{\varphi}_{i}\right|^{2} e^{-2 \bar{\varphi}_{m}} h_{m}^{2} \\
+ \text { constant }
\end{gathered}
$$

clearly, $\bar{\varphi}_{m} \geqq-C$ on $\partial M$, therefore we can chop it off from below as before without increasing the energy. Therefore we can assume that our minimizing sequence $\varphi^{(k)}$ has the property that $\bar{\varphi}_{m} \geqq-C$, which, according to (2.6), implies that

$$
\varphi_{m}^{(k)} \leqq C \text {. }
$$

The new minimizing sequence might lose the lower bound (2.4), but we can chop it off from below and gain back this property easily.

Putting the above together, we have obtained a minimizing sequence $\varphi^{(k)}$ with bounds on $\varphi_{m}^{(k)}$ from above and below ((2.4) and (2.8)). With these bounds, one can easily obtain a minimum by using some standard functional analysis argument.

Remark 2.3. In Theorem 2.1, if there are finite disjoint $n-2$ closed dimensional submanifolds $N_{1}, \ldots, N_{l} \subset M$, one can prove the same result by letting $\rho(x)=\operatorname{dist}\left(x, N_{j}\right)(j=1,2, \ldots, l)$ near $\bigcup_{j=1}^{l} N_{j}$ and smooth, positive elsewhere, also $f_{i}=C_{i}^{j}$ on $N_{j}(i \leqq j \leqq l, 1 \leqq i \leqq m-1)$. The proof is very similar, just perform the chopping off procedure one by one.

\section{Energy Estimates}

Let $\left(\varphi_{1}, \ldots, \varphi_{m}\right)$ be the solution of the Euler-Lagrange equation of (1.3), i.e.

$$
\left\{\begin{array}{l}
\Delta \varphi_{m}=-\frac{1}{h_{m}^{2}} e^{-2 \varphi_{m}} \sum_{i=1}^{m-1}\left|\nabla \varphi_{i}\right|^{2} \\
\operatorname{div}\left(\frac{e^{-2 \varphi_{m}}}{h_{m}^{2}} \nabla \varphi_{i}\right)=0 \quad \text { for } i=1,2, \ldots, m-1
\end{array} \text { on } M \backslash N\right.
$$


with $\left|\varphi_{m}\right| \leqq C$. Note that here we need to use the assumption in Theorem 1 that $\Delta \log h_{m}=0$ outside $N$. In our proof below and the next two sections, we always use $C$ to denote a universal constant, although its actual value may vary in different places. From now on, we fix a point $x_{0}$ in $N \subset M$.

Write $\rho$ to be the distance function from the subspace $N$ with respect to the metric $d s^{2}$. By our assumptions in Theorem 1.1, we have

$$
h_{m}=\rho^{\alpha} e^{u} \quad(\alpha>0)
$$

in a neighborhood of $x_{0}$, say the unit geodesic ball $B_{1}\left(x_{0}\right)$, where $u$ is smooth in $B_{1}\left(x_{0}\right) \backslash N$ such that for any $0<\varepsilon<1$, there exists some positive constant $C(\varepsilon)>0,|\nabla u(x)| \leqq C(\varepsilon) \rho(x)^{\varepsilon-1}, \forall x \in N$.

We denote by $r_{x}(\cdot)$ the distance function on $M$ from $x_{0}$.

Lemma 3.1. The solution $\left(\varphi_{1}, \ldots, \varphi_{m}\right)$ can be extended across $M \cap B_{1}\left(x_{0}\right)$ to be the weak solutions of (3.1) in the sense: for any smooth functions $\psi_{1}, \ldots, \psi_{n}$ with compact support in $B_{1}\left(x_{0}\right)$, we have

$$
\begin{gathered}
\int_{B_{1}(0)}\left\{\nabla \varphi_{m} \nabla \psi_{m}-\frac{1}{h_{m}^{2}} e^{-2 \varphi_{m}} \sum_{i=1}^{m-1}\left|\nabla \varphi_{i}\right|^{2} \psi_{m}\right\} d V=0, \\
\int_{B_{1}}\left\{\nabla \psi_{i} \nabla \varphi_{i}+2 \nabla \varphi_{i}\left(\frac{\nabla h_{m}}{h_{m}}-\nabla \varphi_{m}\right) \psi_{i}\right\} d V=0 \quad i=1,2, \ldots, m-1 .
\end{gathered}
$$

Proof. Outside $N$, the second equation in (3.1) is equivalent to

$$
\Delta \varphi_{i}-2 \nabla \varphi_{i}\left(\frac{\nabla h_{m}}{h_{m}}-\nabla \varphi_{m}\right)=0
$$

Let $\eta$ be a cut-off function from $R^{1}$ into $R^{1}$ satisfying $\eta(t) \equiv 0$ for $t \leqq 1, \eta(t) \equiv 1$ for $t \geqq 2$. $\quad \eta(t) \geqq 0, \quad\left|\eta^{\prime}(t)\right| \leqq 1$. Then the lemma is proved by multiplying $\eta\left(\frac{\log (-\log \rho)}{\log (-\log \varepsilon)}\right) \psi_{j}(1 \leqq j \leqq m)$ to the first equation of (3.1) and (3.4) above, respectively, integrating by parts, and then taking the limit as $\varepsilon$ goes to zero. Note here that we need to use the fact

$$
\int_{0}^{1} \frac{d \rho}{\rho(\log \rho)^{2}}<\infty .
$$

Lemma 3.2. There is a uniform constant $C$ such that for any $x$ in $B_{\frac{1}{2}}\left(x_{0}\right)$,

$$
\int_{B_{\frac{1}{4}}(x)} r_{x}(z)^{-n+2}\left(\left|\nabla \varphi_{m}\right|^{2}+\sum_{i=1}^{m-1} \frac{\left|\nabla \varphi_{i}\right|^{2}}{h_{m}^{2}}\right)(z) d V(z) \leqq C<\infty .
$$

Proof. Let $G_{x}(z)$ be the Green function on $B_{1}\left(x_{0}\right)$ with singularity at $x$ and vanishing on $\partial B_{1}\left(x_{0}\right), \eta$ be the cut-off function defined as above. Put $\lambda=-1+\inf _{M} \varphi_{m}$, then $-\varphi_{m}-\lambda \geqq 1$. For any $\varepsilon>0$, we smooth the Green function as follows:

$$
G_{x}^{\varepsilon}(z)=\frac{1}{\operatorname{Vol}\left(B_{\varepsilon}(z)\right) \int_{B_{\varepsilon}(z)} G_{x}\left(z^{\prime}\right) d z^{\prime}}
$$


Substituting $\psi_{m}$ in $(3.3)$ by $\left(1-\eta\left(4 r_{x}(z)\right)\right)^{2} G_{x}^{\varepsilon}(z)\left(-\varphi_{m}(z)-\lambda\right)$ and using the boundedness of $\varphi_{m}$, we immediately obtain

$$
\begin{aligned}
& \int_{B_{1}(0)}\left\{h_{m}^{-2} \sum_{i=1}^{m-1}\left|\nabla \varphi_{i}\right|^{2}+\left|\nabla \varphi_{m}\right|^{2}\right\}\left(1-\eta\left(4 r_{x}\right)\right)^{2} G_{x}^{\varepsilon}(z) d V(z) \\
& \leqq C\left\{1-\int_{B_{1}(0)} \nabla \varphi_{m} \cdot\left(\varphi_{m}-\lambda\right) \nabla\left((1-\eta)^{2} G_{x}^{\varepsilon}\right) d V\right\} \\
& \leqq C\left\{1+\frac{1}{2} \int_{B_{1}(0)}\left(\varphi_{m}-\lambda\right)^{2} \Delta\left((1-\eta)^{2} G_{x}^{\varepsilon}\right)(z) d V(z)\right\} \\
& \leqq C\left\{1+\frac{1}{2} \int_{B_{1}(0)}\left(\varphi_{m}-\lambda\right)^{2}(1-\eta)^{2} \Delta G_{x}^{\varepsilon} d V\right\} .
\end{aligned}
$$

(Note that $C$ is always a uniform constant.)

But some direct computations show that the last integral is nonpositive when $\varepsilon$ is sufficiently small. Thus our lemma follows from (3.8) by taking the limit as $\varepsilon$ goes to zero and the fact that $G_{x}(z)$ is equivalent to $r_{x}(z)^{-n+2}$ in $B_{\frac{1}{2}}(x)$.

Before we go further, we would like to make a few remarks. By the well-known regularity theory of harmonic maps (cf. [SU1]), we know that $\left(\varphi_{1}, \ldots, \varphi_{m}\right)$ are as smooth as $\left(h_{1}, \ldots, h_{m}\right)$ outside $N \subset M$, or more precisely, the set where $h_{m}>0$.

Lemma 3.3. For any point $x$ in $B_{1}\left(x_{0}\right) \backslash N$, we have

$$
\left|\nabla \varphi_{m}\right|(x) \leqq \frac{C}{\rho(x)},\left|\nabla \varphi_{i}\right|(x) \leqq C_{\rho}(x)^{-1+\alpha},
$$

where $C$ is a uniform constant independent of $\left(\varphi_{1}, \ldots, \varphi_{m}\right)$.

Proof. Recall that $\left(\varphi_{1}, \ldots, \varphi_{m-1}, h_{m} e^{\varphi_{m}}\right)$ define a harmonic map $\Phi$ from $M \backslash N$ into $H^{m}$. Denote by $e=e(\Phi)$ the energy density of this harmonic map. Then by the standard Bochner formula, one finds by using the hyperbolicity of $\mathrm{H}^{m}$,

$$
-\Delta \sqrt{e(\Phi)} \leqq \mu \sqrt{e(\Phi)}
$$

where $\mu$ is a constant depending only on the upper bound of Ricci curvature on $M$.

Then by applying mean-value inequality or direct Moser iteration to (3.10), we obtain $(\bar{\rho}=\rho(x))$

$$
e(\Phi)(x) \leqq \frac{C}{\operatorname{Vol}\left(B \frac{\bar{\rho}}{2}(x)\right)} \int_{\frac{B \bar{\rho}}{2}(x)} e(\Phi)(z) d V(z) .
$$

On the other hand, according to the definition, we have

$$
\begin{aligned}
e(\Phi) & =\left(\sum_{i=1}^{m-1}\left|\nabla \varphi_{i}\right|^{2}\right) h_{m}^{-2} e^{-2 \varphi_{m}}+\left|\nabla \log h_{m}+\nabla \varphi_{m}\right|^{2} \\
& =h_{m}^{-2} e^{-2 \varphi_{m}}\left(\sum_{i=1}^{m-1}\left|\nabla \varphi_{i}\right|^{2}\right)+\left|\frac{\alpha \nabla \rho}{\rho}+\nabla u+\nabla \varphi_{m}\right|^{2} .
\end{aligned}
$$


Thus by the previous lemma and the assumption of $\nabla u$ near $N$ (cf. (3.2)),

$$
\int_{\frac{B \bar{\rho}}{2}(x)} e(\Phi)(z) d V(z) \leqq C \bar{\rho}^{n-2}=C \rho(x)^{n-2} .
$$

It follows

$$
e(\Phi)(x) \leqq C \rho(x)^{-2}
$$

Note that this $C$ may be different from the previous one, but still independent of $x$ in $B_{1}\left(x_{0}\right)$ and $\left(\varphi_{1}, \ldots, \varphi_{m}\right)$. Then (3.9) follows from (3.14) and (3.12).

Without loss of generality, we may assume that $B_{4}\left(x_{0}\right)$ is geodesically convex.

Corollary 3.1. Let $\left(\varphi_{1}, \ldots, \varphi_{m}\right)$ be the solution of (3.1) with $\left|\varphi_{m}\right|$ bounded. Then

$$
\left|\varphi_{i}(x)-\varphi_{i}(\pi(x))\right| \leqq C \rho^{\alpha} \quad i=1,2, \ldots, m-1,
$$

where $\pi$ is the map from $B_{1}\left(x_{0}\right)$ to $N$ defined as follows: for any $x$, there is a unique geodesic $\gamma$ such that $\gamma(0) \in N, \gamma(\rho(x))=x$, and $\gamma(0)$ is perpendicular to $N$ at $\gamma(0)$, then define $\pi(x)=\gamma(0)$.

Proof. Let $\gamma$ be the unique geodesic joining $\pi(x)$ to $x$ with length $\rho(x)$. Then $\left|\gamma^{\prime}\right| \equiv 1$. By the fundamental theorem of calculus,

$$
\begin{aligned}
\varphi_{i}(x)-\varphi_{i}(\pi(x)) & =\int_{0}^{\rho(x)}\left(\frac{d}{d t} \varphi_{i}(\gamma(t))\right) d t=\int_{0}^{\rho(x)} \nabla \varphi_{i}(\gamma(t)) \cdot\left(\gamma^{\prime}(t)\right) d t \\
& \leqq C \int_{0}^{\rho(x)} t^{\alpha-1} d t=\frac{C}{\alpha} \rho^{\alpha}(x),
\end{aligned}
$$

where Lemma 3.3 has been used.

In particular, if $\alpha>1$, then $\varphi_{i}$ must be constant in each connected component of $N$ in $M$.

Proposition 3.1. (Smallness of the normalized energy). Let $\left(\varphi_{1}, \ldots, \varphi_{m}\right)$ be the solution of (3.1) with $\left|\varphi_{m}\right|$ uniformly bounded. Then for any $\varepsilon>0, x$ in $M$, there is a $\sigma_{x}$ between $e^{-3 C / \varepsilon}$ and $\frac{1}{4}$ such that

$$
\sigma_{x}^{-n+2} \int_{B_{\sigma_{x}}(x)}\left(\left|\nabla \varphi_{m}\right|^{2}+\rho^{-2 \alpha} \sum_{i=1}^{m-1}\left|\nabla \varphi_{i}\right|^{2}\right) d V \leqq \varepsilon,
$$

where $C$ is the uniform constant in (3.6).

Proof. Define

$$
f(\sigma)=\frac{1}{\sigma^{n-2}} \int_{B_{\sigma}(x)}\left(\left|\nabla \varphi_{m}\right|^{2}+\rho^{-2 \alpha} \sum_{i=1}^{m-1}\left|\nabla \varphi_{i}\right|^{2}\right) d V
$$


Then

$$
\begin{aligned}
\int_{0}^{\frac{1}{4}} \frac{f(\sigma)}{\sigma} d \sigma= & \int_{0}^{\frac{1}{4}} \sigma^{-n+1}\left(\int_{B_{\sigma}(x)}\left\{\left|\nabla \varphi_{m}\right|^{2}+\rho^{-2 \alpha} \sum_{i=1}^{m-1}\left|\nabla \varphi_{i}\right|^{2}\right\} d V\right) d \sigma \\
= & -(n-2) \int_{0}^{\frac{1}{4}} \frac{d}{d \sigma}\left(\sigma^{-n+2}\right) \int_{B_{\sigma}(x)}\left(\left|\nabla \varphi_{m}\right|^{2}+\rho^{-2 \alpha} \sum_{i=1}^{m-1}\left|\nabla \varphi_{i}\right|^{2}\right) d V \cdot d \sigma \\
= & -\left.(n-2) \sigma^{-n+2} \int_{B_{\sigma}(x)}\left(\left|\nabla \varphi_{m}\right|^{2}+\rho^{-2 \alpha} \sum_{i=1}^{m-1}\left|\nabla \varphi_{i}\right|^{2}\right) d V\right|_{0+} ^{\left.\right|^{\frac{1}{4}}} \\
& +(n-2) \int_{0}^{\frac{1}{4}} \sigma^{-n+2}\left(\int_{\partial B_{\sigma}(x)}\left(\left|\nabla \varphi_{m}\right|^{2}+\rho^{-2 \alpha} \sum_{i=1}^{m-1}\left|\nabla \varphi_{i}\right|^{2}\right) d S\right) d \sigma \\
= & -\left.(n-2) \sigma^{-n+2} \int_{B_{\sigma}(x)}\left(\left|\nabla \varphi_{m}\right|^{2}+\rho^{-2 \alpha} \sum_{i=1}^{m-1}\left|\nabla \varphi_{i}\right|^{2}\right) d V\right|_{0+} ^{\frac{1}{4}} \\
& +(n-2) \int_{B_{\frac{1}{4}}(x)} r_{x}(z)^{-n+2}\left(\left|\nabla \varphi_{m}\right|^{2}+\rho^{-2 \alpha} \sum_{i=1}^{m-1}\left|\nabla \varphi_{i}\right|^{2}\right)(z) d V(z) .
\end{aligned}
$$

By Lemma 3.2 and Lemma 3.3, all last three integrals are uniformly bounded, so it follows

$$
\int_{0}^{\frac{1}{4}} \frac{f(\sigma)}{\sigma} d \sigma \leqq C .
$$

Then the lemma just follows from a simple estimate on the lower bound of the integral in (3.17).

\section{Hölder Estimates}

All the notations in Sect. 3 will be adopted in this section. The aim of this section is to show the Hölder continuity of the solution $\left(\varphi_{1}, \ldots, \varphi_{m}\right)$ of (3.1) at the points of $N$ where $h_{m}$ is of the form (3.2). Such a Hölder estimate will follow from a strengthened version of energy estimate in Proposition 3.1. Usually, this can be accomplished by means of a monotonicity formula, for instance, in the case of harmonic maps. But this required monotonicity formula is not at hand in our case, so we first need to derive it.

Let $\left(\varphi_{1}, \ldots, \varphi_{m}\right)$ be a fixed solution of (3.1) with $\left|\varphi_{m}\right| \leqq C$ as in Sect. 3, and $B_{4}\left(x_{0}\right)$ is a geodesically convex ball at $x_{0}$ in $N$, in which $h_{m}$ can be written as in (3.2). Also, since Theorem 1.1 is local, we may assume that $M=R^{n}$ and $N=R^{n-2} \subset M$.

Lemma 4.1. For any $\beta, 0 \leqq \sigma \leqq \frac{1}{4}, \varepsilon>0$ and $x \in B_{\frac{1}{2}}\left(x_{0}\right) \cap N$, there is the following inequality.

$$
\begin{aligned}
E_{\frac{\sigma}{2}}(x) \leqq & \varepsilon E_{\sigma}(x)+\frac{C}{\varepsilon \sigma^{n}} \int_{B_{\sigma}(x) \backslash B \frac{\sigma}{2}(x)}\left\{\left|\varphi_{m}-\beta\right|^{2}+h_{m}^{-2} e^{-2 \varphi_{m}} \sum_{i=1}^{m-1}\left|\varphi_{i}-\beta_{i}\right|^{2}\right\} d V \\
& +C_{\alpha} \sigma^{1-\alpha}
\end{aligned}
$$


where $C_{\alpha}$ is a constant which is zero if $\alpha \geqq 1$ and we define

$$
E_{\sigma}(x)=\sigma^{-n+2} \int_{B_{\sigma}(x)}\left\{\left|\nabla \varphi_{m}\right|^{2}+h_{m}^{-2} e^{-2 \varphi_{m}} \sum_{i=1}^{m-1}\left|\nabla \varphi_{i}\right|^{2}\right\} d V .
$$

Proof. Let $\eta_{\mu}: R^{1} \rightarrow R^{1}$ be cut-off functions $(\mu>1)$ satisfying:

$$
\begin{gathered}
\eta_{\mu}(t) \equiv 1 \text { for } t \leqq 1 ; \eta_{\mu}(t) \equiv 0 \text { for } t \geqq \mu ; \quad \eta_{\mu}(t) \geqq 0 ; \\
\left|\eta_{\mu}^{\prime}(t)\right| \leqq \frac{1}{\mu-1} ; \quad\left|\eta_{\mu}^{\prime \prime}(t)\right| \leqq\left(\frac{1}{\mu-1}\right)^{2} .
\end{gathered}
$$

There are two cases: (i) $B_{\frac{3}{4} \sigma}(x) \cap N=\varnothing$; (ii) $B_{\frac{3}{4} \sigma}(x) \cap N \neq \varnothing$. Presumably, the first case is easier. Let us consider (ii) and then indicate why (4.1) is also true in case (i). In case $B_{\frac{3}{4} \sigma}(x) \cap N \neq \varnothing$, all $\beta_{i}(i=1,2, \ldots, m-1)$ are zeroes according to our assumption.

We observe that there is $\sigma_{0}$ between $\frac{3}{4} \sigma$ and $\frac{7}{8} \sigma$ such that

$$
\int_{\partial B_{\sigma_{0}(x)}} h_{m}^{-2} e^{-2 \varphi_{m}} \sum_{i=1}^{m-1}\left|\nabla \varphi_{i}\right|^{2} d V \leqq \frac{8}{\sigma_{B_{\frac{7}{8}} \sigma}(x) \backslash B_{\frac{3}{4} \sigma}(x)}{ }_{m} h_{m}^{-2} e^{-2 \varphi_{m}} \sum_{i=1}^{m-1}\left|\nabla \varphi_{i}\right|^{2} d V
$$

and

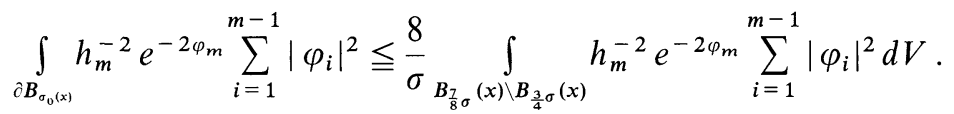

Let $\lambda$ be a positive number $\leqq \frac{1}{16}$, specified later. We call $\left(\varphi_{1}^{*}, \ldots, \varphi_{m}^{*}\right)$ an admissible map if it is in the domain of the functional $F$ (cf. Sect. 1, 2), in particular, for such a map, we have

$$
F\left(\varphi_{1}, \ldots, \varphi_{m}\right) \leqq F\left(\varphi_{1}^{*}, \ldots, \varphi_{m}^{*}\right)
$$

Define

$$
\varphi_{m}^{*}(z)=\left(1-\eta_{\frac{3}{2}}\left(\frac{3}{2 \sigma_{0}} r_{x}(z)\right)\right) \varphi_{m}(z)+\eta_{\frac{3}{2}}\left(\frac{3}{2 \sigma_{0}} r_{x}(z)\right) \beta
$$

In order to define $\varphi_{i}^{*}$ for $1 \leqq i \leqq m-1$, we need to first introduce $\tilde{\varphi}_{i}$ as follows: define $\tilde{\varphi}_{i}(z)$ to be $\varphi_{i}(z)-h_{i}(z)$ for $z \notin B_{\sigma_{0}}(x)$ and $\left(\varphi_{i}-h_{i}\right)\left(x+\frac{\sigma_{0}(z-x)}{z-x}\right)$ for $z$ in $B_{\sigma_{0}}(x)$. In case $\alpha \geqq 1$, we may assume $h_{i}$ are constants in $B_{1}\left(x_{0}\right)$.

Now we define

$$
\varphi_{i}^{*}(z)=\left(1-\eta_{\frac{1}{1-\lambda}}\left(\frac{r_{x}(z)}{(1-\lambda) r_{0}}\right)\right) \tilde{\varphi}_{i}(z)+h_{i}, i=1,2, \ldots, m-1 .
$$

Using the fact that $\rho\left(x+\frac{\sigma_{0}(z-x)}{|z-x|}\right)$ is uniformly equivalent to $\rho(\mathrm{z})$ for $z$ in $B_{\sigma_{0}}(x) \backslash B_{\sigma_{0} / 2}(x)$, one can easily check that $\left(\varphi_{1}^{*}, \ldots, \varphi_{m}^{*}\right)$ is an admissible map, 
moreover, $\varphi_{i}^{*}$ and the derivatives of $\varphi_{i}^{*}(i=1,2, \ldots, m-1)$ coincide with $\varphi_{m}$ and those of $\varphi_{i}$ outside $B_{\sigma_{0}}(x)$, respectively. Therefore, we have

$$
\begin{aligned}
& \int_{B_{\sigma_{0}}(x)}\left\{\left|\nabla \varphi_{m}\right|^{2}+h_{m}^{-2} e^{-2 \varphi_{m}} \sum_{i=1}^{m-1}\left|\nabla \varphi_{i}\right|^{2}\right\} d V \\
\leqq & \int_{B_{\sigma_{0}}(x)}\left\{\left|\nabla \varphi_{m}^{*}\right|^{2}+h_{m}^{-2} e^{-2 \varphi_{m}^{*}} \sum_{i=1}^{m-1}\left|\nabla \varphi_{i}^{*}\right|^{2}\right\} d V \\
= & \int_{B_{\sigma_{0}}(x)}\left\{\left(1-\eta_{\frac{3}{2}}\left(\frac{3}{2 \sigma_{0}} r_{x}\right)\right)\left|\nabla \varphi_{m}\right|^{2}+\frac{9}{4}\left(\eta_{\frac{3}{2}}^{\prime} \frac{1}{\sigma_{0}^{2}}\left|\varphi_{m}-\beta\right|^{2}\right.\right. \\
& -\frac{3}{\sigma_{0}} \eta_{\frac{3}{2}}^{\prime}\left(\frac{3}{2 \sigma_{0}} r_{x}\right) \nabla r_{x} \cdot \nabla \varphi_{m}\left(\varphi_{m}-\beta\right)+h_{m}^{-2} e^{-2 \varphi_{m}^{*}} \\
& \left.\cdot \sum_{i=1}^{m-1}\left|\nabla h_{i}+\left(1-\eta_{\frac{1}{1-\lambda}}\left(\frac{r_{x}}{(1-\lambda) \sigma_{0}}\right)\right) \nabla \tilde{\varphi}_{i}-\frac{\tilde{\varphi}_{i}}{(1-\lambda) \sigma_{0}} \eta_{\frac{1}{1-\lambda}}^{\prime} \cdot \nabla r_{x}\right|^{2}\right\} d V \\
& \int_{B_{\sigma_{0}}(x)}\left\{\left(1-\eta_{\frac{3}{2}}\left(\frac{3 r_{x}}{2 \sigma_{0}}\right)\right)\left|\nabla \varphi_{m}\right|^{2}+\frac{20}{\sigma_{0}^{2}}\left(\left(\eta_{\frac{3}{2}}^{\prime}\right)^{2}+\left|\eta_{\frac{3}{2}}^{\prime \prime}\right|\right)\left|\varphi_{m}-\beta\right|^{2}\right\} d V \\
+ & 4 \int_{B_{\sigma_{0}}(x) \backslash B_{(1-i) \sigma_{0}}(x)} h_{m}^{-2} e^{-2 \varphi_{m}^{*}} \sum_{i=1}^{m-1}\left|\nabla \tilde{\varphi}_{i}\right|^{2} d V \\
+ & \frac{1}{\lambda^{2} \sigma_{0}^{2}} \int_{B_{\sigma_{0}}(z) \backslash B_{(1-\lambda) \sigma_{0}}(x)} h_{m}^{-2} e^{-2 \varphi_{m}^{*}} \sum_{i=1}^{m-1} \tilde{\varphi}_{i}^{2} d V \\
+ & 2 \int_{B_{\sigma_{0}}(x)} h_{m}^{-2} e^{-2 \varphi_{m}^{*}} \sum_{i=1}^{m-1}\left|\nabla h_{i}\right|^{2} d V .
\end{aligned}
$$

In particular, it implies

$$
\begin{aligned}
& \int_{B \frac{3 \sigma_{0}(x)}{4}}\left|\nabla \varphi_{m}\right|^{2} d V+\int_{B_{\sigma_{0}}(x)} h_{m}^{-2} e^{-2 \varphi_{m}} \sum_{i=1}^{m-1}\left|\nabla \varphi_{i}\right|^{2} d V \leqq \frac{200}{\sigma_{0}^{2}} \int_{B_{\sigma_{0}}(x) \backslash \frac{B 3 \sigma_{0}}{4}(x)}\left|\varphi_{m}-\beta\right|^{2} d V \\
& +2 \tilde{C} \int_{\boldsymbol{B}_{\sigma_{0}}(x) \backslash \boldsymbol{B}_{(1-i) \sigma_{0}}(x)} h_{m}^{-2} \sum_{i=1}^{m-1}\left|\nabla \tilde{\varphi}_{i}\right|^{2} d V+\frac{2 \tilde{C}}{\lambda^{2} \sigma_{0_{\sigma_{0}}}^{2}} \int_{\boldsymbol{B}_{(1-\Lambda) \sigma_{0}}(x)} h_{m}^{-2} \sum_{i=1}^{m-1} \tilde{\varphi}_{i}^{2} d V,
\end{aligned}
$$

where $\tilde{C}$ is a constant depending only on the supremum of $\left|\varphi_{m}\right|$. Since $x \in N$ and $\lambda \leqq \frac{1}{16}$, two quantities $h_{m}(z)$ and $h_{m}\left(x+\frac{\sigma_{0}(z-x)}{|z-x|}\right)$ are uniformly equivalent for $z$ in $B_{\sigma_{0}}(x) \backslash B_{(1-\lambda) \sigma_{0}}(x)$, say

$$
C_{1}^{-1} h_{m}(z) \leqq h_{m}\left(x+\frac{\sigma_{0}(z-x)}{|z-x|}\right) \leqq C_{1} h_{m}(z),
$$

where $C_{1}$ is a universal constant. Thus by using

$$
\begin{aligned}
& \int_{\boldsymbol{B}_{\sigma_{0}}(x) \backslash \boldsymbol{B}_{(1-\mathrm{i}) \sigma_{0}}(x)} h_{m}^{-2} \sum_{i=1}^{m-1}\left|\nabla \tilde{\varphi}_{i}\right|^{2} d V \leqq C_{1} \int_{\partial \boldsymbol{B}_{\sigma_{0}}(x)} h_{m}^{-2} \sum_{i=1}^{m-1}\left|\nabla \varphi_{i}\right|^{2} d S \cdot \int_{1-\lambda}^{1} t^{n-1} d t \\
& \leqq \lambda C_{1} \tilde{C} \int_{\partial \boldsymbol{B}_{\sigma_{0}}(x)} h_{m}^{-2} e^{-2 \varphi_{m}} \sum_{i=1}^{m-1}\left|\nabla \varphi_{i}\right|^{2} d V \\
& \leqq 8 \lambda C_{1} \tilde{C} \int_{\boldsymbol{B}_{\sigma_{0}}(x) \backslash B_{\frac{3}{4} \sigma_{0}}(x)} h_{m}^{-2} e^{-2 \varphi_{m}} \sum_{i=1}^{m-1}\left|\nabla \varphi_{i}\right|^{2} d V .
\end{aligned}
$$


Similarly,'we also have

$$
\int_{B_{\sigma_{0}}(x) \backslash B_{(1-i) \sigma_{0}}(x)} h_{m}^{-2} \sum_{i=1}^{m-1} \tilde{\varphi}_{i} d V \leqq 8 \lambda C_{1} \tilde{C} \int_{B_{\frac{\eta^{\sigma}}{\sigma}}(x) \backslash B_{\frac{3}{4} \sigma}(x)} h_{m}^{-2} e^{-2 \varphi_{m}} \sum_{i=1}^{m-1} \varphi_{i}^{2} d V .
$$

Combining (4.9), (4.11) and (4.12), we obtain

$$
\begin{aligned}
& \int_{\frac{3 \sigma_{0}}{4}(x)}\left|\nabla \varphi_{m}\right|^{2} d V+\int_{B_{\sigma_{0}}(x)} h_{m}^{-2} e^{-2 \varphi_{m}} \sum_{i=1}^{m-1}\left|\nabla \varphi_{i}\right|^{2} d V \leqq \frac{200}{\sigma_{0}^{2}} \int_{B_{\sigma_{0}}(x) \backslash B_{\frac{3 \sigma_{0}}{4}}(x)}\left|\varphi_{m}-\beta\right|^{2} d V \\
& +16 \lambda \tilde{C}^{2} C_{1}\left\{\int_{B_{\frac{1}{\delta} \sigma}(x) \backslash B_{\frac{3}{4} \sigma}(x)} h_{m}^{-2} e^{-2 \varphi_{m}} \sum_{i=1}^{m-1}\left|\nabla \varphi_{i}\right|^{2} d V\right. \\
& \left.+\frac{1}{\lambda^{2} \sigma_{0}^{2}} \int_{B_{\bar{B}_{\sigma}}(x) \backslash B_{\frac{3}{4} \sigma}(x)} h_{m}^{-2} e^{-2 \varphi_{m}} \sum_{i=1}^{m-1} \varphi_{i}^{2} d V\right\} \text {. }
\end{aligned}
$$

Now we choose $\lambda$ such that $16 \lambda \tilde{C}^{2} C_{1} \leqq \varepsilon$. Then there is a uniform constant $C_{0}$ such that

$$
\begin{aligned}
& \int_{B_{\frac{3}{4} \sigma_{0}}(x)}\left\{\left|\nabla \varphi_{m}\right|^{2}+h_{m}^{-2} e^{-2 \varphi_{m}} \sum_{i=1}^{m-1}\left|\nabla \varphi_{i}\right|^{2}\right\} d V \\
& \leqq \varepsilon E_{\sigma}(x)+\frac{C_{0}}{\varepsilon \sigma^{2}}\left\{\int_{B_{\sigma}(x) \backslash B_{3^{\prime} \sigma}(x)}\left(\left|\varphi_{m}-\beta\right|^{2}+h_{m}^{-2} e^{-2 \varphi_{m}} \sum_{i=1}^{m-1}\left|\nabla \varphi_{i}\right|^{2}\right) d V\right\} .
\end{aligned}
$$

Note that $\frac{3}{4} \sigma \leqq \sigma_{0} \leqq \frac{7 \sigma}{8}$, so $\frac{3 \sigma_{0}}{4} \geqq \frac{1}{2} \sigma, \sigma_{0} \leqq \sigma$, so the lemma follows from the above inequality.

Lemma 4.2. For any $\beta, 0<\sigma \leqq \frac{1}{4}, \varepsilon>0$ and $x \in B_{\frac{1}{2}}\left(x_{0}\right)$, there is the following inequality:

$$
\begin{aligned}
E_{\frac{\sigma}{2}}(x) \leqq & \varepsilon E_{\sigma}(x)+\frac{C}{\varepsilon \sigma^{n}} \int_{B_{\sigma}(x) \backslash B_{\frac{\sigma}{\xi}}(x)}\left\{\left|\varphi_{m}-\beta\right|^{2}+h_{m}^{-2} e^{-2 \varphi_{m}} \sum_{i=1}^{m-1}\left|\varphi_{i}-\beta_{i}\right|^{2}\right\} d V \\
& +C_{\alpha} \sigma^{1-\alpha},
\end{aligned}
$$

where $\beta_{1}, \ldots, \beta_{m-1}$ are any constants if $B_{\sigma / 5}(x) \cap N=\varnothing$; zeroes otherwise, $C_{\alpha}$ is a constant which is zero if $\alpha \geqq 1$ and we define

Proof. There are two cases: (i) $B_{\sigma / 5}(x) \cap N \neq \varnothing$; (ii) $B_{\sigma / 5}(x) \cap N=\varnothing$. In the first case, let $y$ be a point in $B_{\sigma / 5}(x) \cap N$, then

$$
B_{\frac{\sigma}{8}}(x) \subset B_{\frac{3}{8} \sigma}(y), \quad B_{\frac{3}{4} \sigma}(y) \subset B_{\sigma}(x) .
$$

Applying Lemma 4.1 with $\varepsilon$ replaced by $\varepsilon^{\prime}=4^{-n+2} \varepsilon$, we have

$$
\begin{aligned}
E_{\frac{\sigma}{8}}(x) \leqq & 3^{n-2}\left\{\varepsilon^{\prime} E_{\frac{3 \sigma}{4}}(y)+\frac{C}{\varepsilon^{\prime} \sigma^{n}} \int_{B_{\frac{3}{4}} \sigma} \int_{i=\frac{3}{\frac{3}{8}}(y)}\left\{\left|\varphi_{m}-\beta\right|^{2}+h_{m}^{-2} e^{-2 \varphi_{m}} \sum_{i=1}^{m-1}\left|\varphi_{i}-\beta_{i}\right|^{2}\right\} d V\right. \\
& \left.+C_{\alpha} \sigma^{1-\alpha}\right\} \\
\leqq & \varepsilon E_{\sigma}(x)+\frac{C}{\varepsilon \sigma^{n}} \int_{B_{\sigma}(x) \backslash B_{\frac{\delta}{8}}(x)}\left\{\left|\varphi_{m}-\beta\right|^{2}+h_{m}^{-2} e^{-2 \varphi_{m}} \sum_{i=1}^{m-1}\left|\varphi_{i}-\beta_{i}\right|^{2}\right\} d V \\
& +C_{\alpha} \sigma^{1-\alpha} .
\end{aligned}
$$


In the second case, we may simply take

$$
\varphi_{j}^{*}(z)=\left(1-\eta_{\frac{4}{3}}\left(\frac{8 r_{x}}{\sigma}\right)\right) \varphi_{j}+\eta_{\frac{4}{3}}\left(\frac{8 r_{x}}{\sigma}\right) \beta_{j}, \quad 1 \leqq j \leqq m
$$

as comparison functions in the derivation of (4.8) and proceed as in the proof of Lemma 4.1; we can still obtain (4.12).

Next, we give a weighted Poincaré inequality. It should be well-known.

Lemma 4.3. Fix $0<\sigma<\frac{1}{4}$, $x$ in $B_{1}\left(x_{0}\right)$ with $B_{\frac{\sigma}{4}}(x) \cap N \neq \varnothing$. Then

$$
\int_{B_{\sigma}(x) \backslash B_{\frac{1}{2}}(x)} \frac{\psi^{2}}{\rho^{2 \alpha+2}} d V \leqq \gamma \int_{B_{\sigma}(x) \backslash B_{\frac{\sigma}{2}}(x)} \frac{|\nabla \psi|^{2}}{\rho^{2 \alpha}} d V
$$

for any $C^{1}$ function $\psi$ in $B_{\sigma}(x)$ vanishing on $B_{\sigma}(x) \cap N$, where $\gamma$ is a universal constant possibly depending only on $n$.

Proof. First let us make a few observations: (i) $d s^{2}$ is very close to the Euclidean one in $B_{1}\left(x_{0}\right)$ by the assumption at the beginning of Sect. 3 . Therefore, it suffices to show (4.14) in case of Euclidean space with $N$ being a subspace; (ii) (4.14) is invariant under scaling, so we may take $\sigma$ to be 2 .

Choose Euclidean coordinates $\left(x_{1}, \ldots, x_{n}\right)$ such that $x=(0, \ldots, 0)$ and $N$ is defined by $x_{n-1}=0, x_{n}=\mu>0$. Since $B_{\frac{1}{2}}(x) \cap N \neq \varnothing, \mu<\frac{1}{2}$. Now $\rho=\sqrt{x_{n-1}^{2}+\left(x_{n}-\mu\right)^{2}}$. Let $\tilde{r}=\sqrt{x_{1}^{2},+\ldots+x_{n-2}^{2}}, \tilde{\rho}=\sqrt{\tilde{r}^{2}+x_{n-1}^{2}+x_{n}^{2}}$.

Let $\eta$ be a function on $B_{2}(x) \backslash B_{1}(x)$ satisfying: $\eta \equiv 1$ if $\tilde{r} \leqq \frac{1}{4} ; \eta \equiv 0$ if $\tilde{r} \geqq \frac{1}{2}$; $|\nabla \eta| \leqq 10$. Then $\eta \psi$ vanishes outside $\tilde{r} \leqq \frac{1}{2}$, so the standard Poincare inequality implies: for a uniform constant $C$,

$$
\begin{gathered}
\int_{B_{2}(x) \backslash B_{1}(x)}(\eta \psi)^{2} d V \leqq C \int_{B_{2}(x) \backslash B_{1}(x)}|\nabla(\eta \psi)|^{2} d V \\
\leqq 20 C\left(\int_{B_{2}(x) \backslash B_{1}(x)}|\nabla \psi|^{2} d V+\int_{\substack{B_{2}(x) \backslash B_{1}(x) \\
\frac{1}{2} \geqq \dot{r} \geqq \frac{1}{4}}} \psi^{2} d V\right) .
\end{gathered}
$$

However, in case $\tilde{r} \leqq \frac{1}{4}, 3 \geqq \rho \geqq \frac{1}{4}$. It follows that for some $\tilde{C}$,

$$
\int_{\substack{B_{2}(x) \backslash B_{1}(x) \\ \tilde{r} \leqq \frac{1}{4}}} \frac{\psi^{2}}{\rho^{2 \alpha+2}} d V \leqq \tilde{C}\left(\int_{B_{2}(x) \backslash B_{1}(x)} \frac{|\nabla \psi|^{2}}{\rho^{2 \alpha}} d V+\int_{\substack{B_{2}(x) \backslash B_{1}(x) \\ \tilde{r} \leqq \frac{1}{4}}} \frac{\psi^{2}}{\rho^{2 \alpha+2}} d V\right) .
$$

Therefore, to prove (4.14), it suffices to show

$$
\int_{\substack{\boldsymbol{B}_{2}(x) \backslash \boldsymbol{B}_{1}(x) \\ \tilde{\boldsymbol{r}} \geqq \frac{1}{4}}} \frac{\psi^{2}}{\rho^{2} \propto C^{+2}} d V \leqq C^{\prime} \int_{\boldsymbol{B}_{2}(x) \backslash \boldsymbol{B}_{1}(x)} \frac{|\nabla \psi|^{2}}{\rho^{2 \alpha}} d V .
$$

Using the spherical coordinates for $\left(x_{1}, \ldots, x_{n-2}\right)$ and polar coordinates $(\rho, \theta)$ for $\left(x_{n-1}, x_{n}-\mu\right)$, one can easily reduce (4.17) to the following inequality:

$$
\begin{gathered}
\int_{1 \leqq \tilde{r}^{2}+\left(\rho+\mu \sin _{\tilde{r} \geqq \frac{1}{4}}+\mu^{2} \cos ^{2} \theta \leqq 4\right.} \tilde{r}^{n-3} \rho^{-2 \alpha-1}|\psi|^{2} d \tilde{r} d \rho \\
\leqq C^{\prime} \underset{1 \leqq \tilde{r}^{2}+(\rho+\mu \sin \theta)^{2}+\mu^{2} \cos ^{2} \theta \leqq 4}{\iint^{n-3} \rho^{-2 \alpha-1}|\nabla \psi|^{2} d \tilde{r} d \rho,}
\end{gathered}
$$


where $0 \leqq \theta \leqq 2 \pi$. Note that using $\mu \leqq \frac{1}{2}$ so we may simply write $\mu$ for $\mu \sin \theta$ and put $b=4-\mu^{2} \cos ^{2} \theta \leqq 4, a=1-\mu^{2} \cos ^{2} \theta \geqq \frac{3}{4}$, then $b-a=3$. Write $t=\rho+\mu$, then $\rho=t-\mu$; we obtain the equivalent form of (4.18),

$\iint_{\substack{a \leqq r^{2}+t^{2} \leqq b \\ \tilde{r} \geqq \frac{1}{4}}} \tilde{r}^{n-3}|t-\mu|^{-2 \alpha-1}|\psi|^{2} d \tilde{r} d t \leqq \gamma \iint_{a \leqq r^{2}+t^{2} \leqq b} \tilde{r}^{n-3}|t-\mu|^{-2 \alpha+1}|\nabla \psi|^{2} d \tilde{r} d t$.

Using the polar coordinates $(\tilde{\rho}, \tilde{\theta})$ of $(\tilde{r}, t)$, we can easily see that (4.19) follows from the inequalities:

$$
\begin{aligned}
& \int_{\tilde{\rho} \cos \tilde{\theta} \geqq \frac{1}{4}}(\cos \tilde{\theta})^{n-3}\left|\sin \tilde{\theta}-\frac{\mu}{\tilde{\rho}}\right|^{-2 \alpha-1}|\psi|^{2} d \tilde{\theta} \\
& \leqq \gamma \int_{\tilde{\rho} \cos \tilde{\theta} \geqq \frac{1}{4}}(\cos \tilde{\theta})^{n-3}\left|\sin \tilde{\theta}-\frac{\mu}{\tilde{\rho}}\right|^{-2 \alpha+1}\left|\frac{d \psi}{d \tilde{\theta}}\right|^{2} d \tilde{\theta},
\end{aligned}
$$

where $\frac{3}{4} \leqq \tilde{\rho} \leqq 4,|\mu| \leqq \frac{1}{2}$. Note that $\psi$ vanishes at those points with $\sin \tilde{\theta}=\mu / \tilde{\rho}$, which are indeed in the path $\left\{\tilde{\rho} \cos \tilde{\theta} \geqq \frac{1}{4}\right\}$.

The inequality (4.20) can be proved by using integration by parts, the Schwartz inequality and the fact that $\cos \tilde{\theta}$ is bounded from below.

Corollary 4.1. For $1<\sigma<\frac{1}{4}, x$ in $B_{1}\left(x_{0}\right)$ with $B_{\frac{\sigma}{4}}\left(x_{0}\right) \cap N \neq \varnothing$. Then

$$
\int_{B_{\sigma}(x)} \frac{\psi^{2}}{\rho^{2 \alpha+2}} d V \leqq \gamma \int_{B_{\sigma}(x)} \frac{|\nabla \psi|^{2}}{\rho^{2 \alpha}} d V
$$

for any $C^{1}$ function $\psi$ in $B_{\sigma}(x)$ vanishing on $B_{\sigma}(x) \cap N$, where $\tilde{\gamma}$ is a universal constant depending only on $n$.

Proof. Choose a point $\tilde{x}$ in $B_{\sigma / 4}(x) \cap N$, then

$$
B_{\frac{\sigma}{2}}(x) \subset B_{\frac{3}{4} \sigma}(\tilde{x}) \subset B_{\sigma}(x) .
$$

According to Lemma 4.3, we obtain

$$
\begin{aligned}
& \int_{B_{\sigma}(x) \backslash B_{\frac{\sigma}{2}(x)}} \frac{\psi^{2}}{\rho^{2 \alpha+2}} d V \leqq \gamma \int_{B_{\sigma}(x) \backslash B \frac{\sigma}{2}(x)} \frac{|\nabla \psi|^{2}}{\rho^{2 \alpha}} d V
\end{aligned}
$$

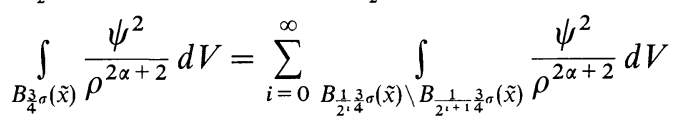

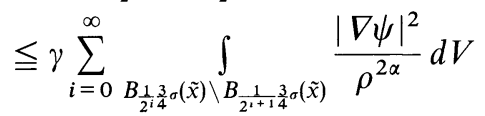

$$
\begin{aligned}
& =\int_{B_{\frac{3}{4} \sigma(\tilde{x})}} \frac{\psi^{2}}{\rho^{2 \alpha}} d V
\end{aligned}
$$

therefore (4.21) follows with $\tilde{\gamma}=2 \gamma$.

Proposition 4.1. There are $\varepsilon_{0}$ and $\lambda_{0}$, independent of $x$ in $B_{1 / 2}\left(x_{0}\right)$, such that whenever

$$
E_{\sigma}(x) \leqq \varepsilon_{0}^{2}, \quad 0<\sigma \leqq \frac{1}{4}, \quad x \in B_{\frac{1}{2}}\left(x_{0}\right),
$$


we have

$$
E_{\lambda_{0} \sigma}(x) \leqq \frac{1}{2} E_{\sigma}(x)
$$

Proof. We will prove this proposition in case $\alpha \geqq 1$. The proof of the remaining case $\alpha<1$ is completely identical except that we use $\varphi_{i}-h_{i}$ in place of $\varphi_{i}$ $(i=1,2, \ldots, m-1)$. Since $h_{i}$ are assumed to be smooth across $N$ for $i=1,2, \ldots, m-1$, this modification won't affect any argument below.

In case $\alpha \geqq 1$, we may assume that $\varphi_{i}$ are zero along $N$ for $i=1,2, \ldots, m-1$ (cf. Sect. 2).

We prove (4.23) by contradiction. Suppose that this proposition is not true. Then there are sequences of $\left\{x_{i}\right\}_{i \geqq 1}$ in $B_{1 / 2}\left(x_{0}\right),\left\{\varepsilon_{i}\right\}_{i \geqq 1}$, and $\left\{\sigma_{i}\right\}_{i \geqq 1}$ such that $0<\sigma_{i} \leqq \frac{1}{4}$, $\lim _{i \rightarrow \infty} \varepsilon_{i}=0$, and

$$
\begin{gathered}
\varepsilon_{i}^{2}=E_{\sigma_{i}}\left(x_{i}\right), \\
E_{\lambda_{0} \sigma_{i}}\left(x_{i}\right)>\frac{1}{2} E_{\sigma_{i}}\left(x_{i}\right)=\frac{1}{2} \varepsilon_{i}^{2},
\end{gathered}
$$

where $\lambda_{0}$ is a small number, determined later.

For simplicity of notations, we assume that $B_{1}\left(x_{0}\right)$ is the Euclidean ball in $R^{n}$ with $x_{0}$ being the origin, $d s^{2}$ is just the Euclidean metric and $N=R^{n-2} \subset R^{n}$. The proof in general case is completely analogous.

By taking a subsequence of $\left\{x_{i}\right\}$, we may assume that $x_{i}$ converge to a point $\tilde{x}$ in $B_{1 / 2}\left(x_{0}\right)$. Since the solution $\left(\varphi_{1}, \ldots, \varphi_{m}\right)$ has been known to be regular outside $N$, such a point $\tilde{x}$ must be in $B_{1 / 2}\left(x_{0}\right) \cap N$.

First let us consider the case: there is a subsequence of $\left\{x_{i}\right\}$ such that for any $x_{i}$ in this sequence, $B_{\sigma_{i} / l_{0}}\left(x_{i}\right) \cap N=\varnothing$, where $l_{0}$ will be determined later prior to the determination of $\lambda_{0}$. We remark that this $l_{0}$ will be independent of $x$ in $B_{1 / 2}\left(x_{0}\right)$. For simplicity, assume $\left\{x_{i}\right\}$ is just this sequence. Then $\rho(x) \geqq \sigma_{i} / 2 l_{0}$ for $y$ in $B_{\sigma_{i} / 2 l_{0}}\left(x_{i}\right)$. Define functions on $B_{1}(0) \subset R^{n}$ as follows: $i \geqq 1$,

$$
\begin{aligned}
\psi_{j}^{i}(x) & =\frac{1}{\rho\left(x_{i}\right)^{\alpha} \varepsilon_{i}}\left(\varphi_{j}\left(x_{i}+\sigma_{i} x\right)-\bar{\varphi}_{j}^{i}\right), \quad x \in B_{1}(0), \quad j=1,2, \ldots, m, \\
\psi_{m}^{i}(x) & =\frac{1}{\varepsilon_{i}}\left(\varphi_{m}\left(x_{i}+\sigma_{i} x\right)-\bar{\varphi}_{m}^{i}\right), \quad x \in B_{1}(0),
\end{aligned}
$$

where $\bar{\varphi}_{j}^{i}, \bar{\varphi}_{m}^{i}$ are the averages of $\varphi_{j}, \varphi_{m}$ over $B_{\sigma_{i} / 2 l_{0}}\left(x_{i}\right)$, respectively.

Now $E_{\sigma_{i}}\left(x_{i}\right)=\varepsilon_{i}^{2}$ implies that

$$
\int_{B \frac{1}{2 t_{0}}(0)}\left|\nabla \psi_{j}^{i}\right|^{2} d V \leqq 4 \quad \text { for } j=1,2, \ldots, m ; \quad i \geqq 1 .
$$

By taking the subsequence, we may assume that $\psi_{j}^{i}$ converge weakly to $\psi_{j}$ in $H_{2}^{1}$-norms. Note that we need to use the fact that the averages of $\psi_{j}^{i}$ are zeroes in $B_{1 / 2 l_{0}}(0)$. Moreover, the standard argument shows that $\psi_{j}^{i}$ converge strongly to $\psi_{j}$ in $L^{p}$-norms for any $p<\frac{2 n}{n-2}$ in case $n>2$ or $p<\infty$ in case $n=2$. Using (3.1), one can easily show that in the sense of distribution,

$$
\left\{\begin{array}{l}
\Delta \psi_{m}=0 \\
\Delta \psi_{j}=2 \nabla \psi_{j} \cdot \nabla \theta, \quad j=1,2, \ldots, m-1,
\end{array}\right.
$$


where $\theta$ is a smooth, uniformly bounded function. Then we conclude from the standard elliptic theory (cf. [GT]) that

$$
\left|\psi_{j}(x)-\psi_{j}(0)\right| \leqq C_{1}|x| \text { for } x \text { in } B_{\frac{1}{2 l_{0}}}(0) \quad j=1,2, \ldots, m .
$$

Let $\lambda_{0}$ be smaller than $\frac{1}{32}$. Applying (4.1) with $\varepsilon=\frac{1}{8}$ and $\varepsilon_{i} \rho\left(x_{i}\right)^{\alpha} \psi_{j}(0)$ as $\beta_{j}$ for $j \leqq m-1$, and $\varepsilon_{i} \psi_{m}(0)$ as $\beta_{m}$, we obtain

$$
\begin{aligned}
\varepsilon_{i}^{2}= & E_{\lambda_{0} \sigma_{i}}\left(x_{i}\right) \leqq \frac{1}{8} E_{8 \lambda_{0} \sigma_{i}}\left(x_{i}\right)+\frac{C}{\left(\lambda_{0} \sigma_{i}\right)^{n}}{ }_{B_{8 \lambda_{0} \sigma_{i}}\left(x_{i}\right) \backslash B_{\lambda_{0_{i}}}\left(x_{i}\right)}\left\{\left|\varphi_{m}-\varepsilon_{i} \psi_{m}(0)\right|^{2}\right. \\
& \left.+\rho^{-2 \alpha} \sum_{j=1}^{m-1}\left|\varphi_{j}-\varepsilon_{i} \rho\left(x_{i}\right)^{\alpha} \psi_{j}(0)\right|^{2}\right\} d V \\
\leqq & \frac{1}{8 l} E_{8^{2} \lambda_{0} \sigma_{i}}\left(x_{i}\right)+8 C \sum_{s=1}^{l} \frac{1}{8^{s}\left(8^{s} \lambda_{0} \sigma_{i}\right)^{n}}{ }_{B_{s^{s} \lambda_{0} \sigma_{i}}\left(x_{i}\right) \backslash B_{8}{ }^{s-1}{ }_{\lambda_{0} \sigma_{i}}\left(x_{i}\right)}\left\{\left|\varphi_{m}-\varepsilon_{i} \psi_{m}(0)\right|^{2}\right. \\
& \left.+\rho^{-2 \alpha} \sum_{j=1}^{m-1}\left|\varphi_{j}-\varepsilon_{i} \rho\left(x_{i}\right)^{\alpha} \psi_{j}(0)\right|^{2}\right\} d V
\end{aligned}
$$

(choose $l$ such that $8^{l} \lambda_{0} \leqq \frac{1}{8}$ )

$$
\begin{aligned}
& \leqq \frac{1}{8^{l}} E_{8^{l} \lambda_{0} \sigma_{i}}\left(x_{i}\right) \\
& \left.+2^{6 \alpha+3} C \varepsilon_{i}^{2} \sum_{s=1}^{l} \frac{1}{8^{s}\left(8^{s} \lambda_{0}\right)^{n}} \int_{B_{8} \lambda_{\lambda_{0}}(0) \backslash B_{8}^{s-1} \lambda_{\lambda_{0}}(0)} \sum_{j=1}^{m}\left|\psi_{j}^{i}-\psi_{j}(0)\right|^{2}\right) d V \\
& \leqq \frac{1}{8^{l}} E_{8^{l} \lambda_{0} \sigma_{i}}\left(x_{i}\right) \\
& +2^{6 \alpha+4} C \varepsilon_{i}^{2} \sum_{s=1}^{l} \frac{1}{8^{s}\left(8^{s} \lambda_{0}\right)^{n}} \int_{B_{8^{s} \lambda_{0}}(0) \backslash B_{B^{s-1} \lambda_{0}}(0)}\left(\sum_{j=1}^{m}\left|\psi_{j}^{i}-\psi_{j}\right|^{2}\right. \\
& \left.+\left|\psi_{j}-\psi_{j}(0)\right|^{2}\right) d V \\
& \leqq \frac{1}{8^{l}} E_{8^{l} \lambda_{0} \sigma_{i}}\left(x_{i}\right) \\
& +C_{2} \varepsilon_{i}^{2} \lambda_{0}^{2} \sum_{s=1}^{l} \frac{1}{8^{s}}+2^{6 \alpha+4} C \varepsilon_{i}^{2} \frac{1}{\left(\lambda_{0}\right)^{n}}{ }_{B_{8}{ }^{1} \lambda_{0}(0) \backslash B_{\alpha_{0}}(0)} \sum_{j=1}^{m}\left|\psi_{j}^{i}-\psi_{j}\right|^{2} d V,
\end{aligned}
$$

where $C_{2}$ depends only on $C$ and $C_{1}$. Note that $C$ always denotes a uniform constant, although its value may vary in different places.

Now we choose $\lambda_{0}, l$ such that $C_{2} \lambda_{0}^{2} \leqq 1, \frac{1}{4 l_{0}} \leqq 8^{l} \lambda_{0} \leqq \frac{1}{2 l_{0}}$ and $\frac{1}{8 l}\left(4 l_{0}\right)^{n-2} \leqq \frac{1}{8}$, then take $i$ sufficiently large such that the last term in (4.30) is less than $\frac{1}{4}$. Here we use the convergence of $\psi_{j}^{i}$ to $\psi_{j}(j=1,2, \ldots, m)$. Therefore, we have

$$
E_{\lambda_{0} \sigma_{i}}\left(x_{i}\right) \leqq \frac{1}{2} \varepsilon_{i}^{2} .
$$

It contradicts to the inequality (4.25). 
Next, we may assume that $B_{\frac{1}{l_{o}}}\left(x_{i}\right) \cap N \neq \varnothing$ for all $i$. This time we define

$$
\begin{aligned}
\psi_{j}^{i}(x) & =\frac{1}{\sigma_{i}^{\alpha} \varepsilon_{i}} \varphi_{j}\left(x_{i}+\sigma_{i} x\right), \quad j=1,2, \ldots, m-1, \\
\psi_{m}^{i}(x) & =\frac{1}{\varepsilon_{i}}\left(\varphi_{m}\left(x_{i}+\sigma_{i} x\right)-\bar{\varphi}_{m}\right),
\end{aligned}
$$

where $x$ is in $B_{1}(0)$ and $\bar{\varphi}_{m}$ denotes the average of $\varphi_{m}$ over $B_{\sigma_{i}}\left(x_{i}\right)$.

Under the transformations: $x \rightarrow x_{i}+\sigma_{i} x$ in $R^{n}$, the preimages of $N$ are $N_{i}$ parallel to the subspace $R^{n-2} \subset R^{n}$ and in the distance $\pi\left(x_{i}\right) / \sigma_{i}$, where $\pi$ is the orthogonal projection from $R^{n}$ onto $R^{n-2}$. Since $B_{\sigma_{i} / l_{0}}\left(x_{i}\right) \cap N \neq \varphi$, we have $\left|\pi\left(x_{i}\right) / \sigma_{i}\right| \leqq 1 / l_{0} \leqq \frac{1}{4}$. Thus we may assume that $N_{i}$ converge to an affine subspace $N_{\infty}$ in $R^{n}$ within the distance $1 / l_{0}$ from $R^{n-2}$. Let $\rho_{i}, \rho_{\infty}$ be the distance from $N_{i}$, $N_{\infty}$, respectively. Then

$$
\int_{B_{1}(0)}\left\{\left|\nabla \psi_{m}^{i}\right|^{2}+2^{2 \varphi_{m}\left(x_{i}+\sigma_{i} x\right)}\left(\sum_{j=1}^{m-1}\left|\nabla \psi_{j}^{i}\right|^{2}\right) \rho_{i}^{-2 \alpha}\right\} d V=1 .
$$

Note that $\varphi_{m}$ is uniformly bounded. Apply the Corollary 4.1, we have

$$
\int_{B_{1}(0)}\left\{\left|\psi_{m}^{i}\right|^{2}+\rho_{i}^{-2 \alpha-2} \sum_{j=1}^{m-1}\left|\psi_{j}^{i}\right|^{2}\right\} d V \leqq C .
$$

Recall our convention that $C$ always denotes a uniform constant. As in the previous case, these $\psi_{m}^{i}, \psi_{j}^{i}(1 \leqq j \leqq m-1)$ converge to $\psi_{m}, \psi_{j}$ in $H_{2}^{1}$-norm and $H_{2, \rho^{\alpha}}^{1}$ norms respectively, where

$$
\|\psi\|_{H_{2} \frac{1}{2}}=\int_{B_{1}(0)}\left(\frac{|\nabla \psi|^{2}}{\rho^{2 \alpha}}+\frac{\psi^{2}}{\rho^{2 \alpha+2}}\right) d V .
$$

Moreover, the functions $\tilde{\psi}_{m}=\psi_{m}$ and $\tilde{\psi}_{j}=\frac{1}{\rho_{\infty}^{\alpha}} \psi_{j}$ satisfy the following equations in the weak sense:

$$
\begin{gathered}
\Delta \tilde{\psi}_{m}=0 \\
\Delta \tilde{\psi}_{j}=2 \nabla \tilde{\psi}_{j} \nabla \theta_{j}+\frac{\alpha \tilde{\psi}_{j}}{\rho_{\infty}}\left(\frac{\alpha+1}{\rho_{\infty}}-\nabla \theta_{j} \nabla \rho_{\infty}\right),
\end{gathered}
$$

where $\theta_{j}$ are smooth outside $N_{\infty}$ in $B_{1}(0)$ and $\left|\nabla \theta_{j}\right| \leqq C_{\delta}^{\prime} \rho_{\infty}^{-\delta}$ for any $\delta<1$ and some constant $C_{\delta}^{\prime}$. So we still have the estimate (4.29) for this $\tilde{\psi}_{m}$. Also, these $\tilde{\psi}_{j}$ are uniformly bounded outside the set

$$
U=\left\{\rho_{\infty}^{-1}>2 \max \max _{1 \leqq j \leqq m-1}\left\{\nabla \theta_{j} \nabla \rho_{\infty}(x) \mid x \in B_{1}(0)\right\}\right\},
$$

since $\rho_{\infty}$ is smooth here. On the other hand, for any $x$ in $U$,

$$
\left(\frac{\alpha+1}{\rho_{\infty}}-\nabla \theta_{j} \nabla \rho_{\infty}\right)(x)>0 \text {. }
$$


Applying Moser's iteration (cf. [GT], Chap. 8), one can show

$$
\sup _{B_{\frac{1}{2}}(0)}\left|\tilde{\psi}_{j}\right| \leqq C\left(1+\int_{B_{1}(0)}\left|\tilde{\psi}_{j}\right|^{2} d V\right)^{\frac{1}{2}}, \quad j=1,2, \ldots, m-1 .
$$

In particular, these $\tilde{\psi}_{j}$ are uniformly bounded, or equivalently, $\rho_{\infty}^{-\alpha} \psi_{j}$ are uniformly bounded. It also implies that $\psi_{j}$ vanishes along $N_{\infty} \cap B_{1}(0)$.

Next, we need the following lemma, whose proof will be given later.

Lemma 4.4. Let $v$ be a smooth function outside $N_{\infty} \cap B_{1}(0)$ and $|\nabla v| \leqq C^{\prime} \rho_{\infty}^{-\beta}$ with $\beta<1, f$ be a smooth function in $B_{1}(0)$ with $\max _{B_{1}(0)}\left(\rho_{\infty}^{2-2 \alpha}|f|\right) \leqq C^{\prime}$. Then for any $\varepsilon>0$, there is a uniform constant $C_{\varepsilon}$, depending only on $C^{\prime}, \beta, \varepsilon$, such that if $u$ is a solution of the equation

$$
\Delta u=2 \nabla u\left(\frac{\alpha \Delta \rho_{\infty}}{\rho_{\infty}}+\nabla v\right)+f \text { in } B_{1}(0)
$$

and $|u|_{C^{0}\left(\hat{\partial} B_{2 / 3}(0)\right)} \leqq C^{\prime},\left.u\right|_{N_{\infty}} \equiv 0$, then

$$
|u(x)| \leqq C_{\varepsilon} \rho_{\infty}^{2 \alpha-2 \varepsilon}(x) \quad \text { for } x \text { in } B_{\frac{1}{2}}(0) .
$$

Applying the lemma to $\psi_{j}(j=1,2, \ldots, m-1)$ with $\varepsilon=\alpha / 4$, we obtain

$$
\begin{gathered}
\left|\psi_{j}(x)\right| \leqq C \rho_{\infty}(x)^{\frac{3 \alpha}{2}}, \quad x \in B_{\frac{1}{2}}(0) \\
j=1,2, \ldots, m-1 .
\end{gathered}
$$

Here, as usual, $C$ denotes a constant independent of $\psi_{j}$ and $x$.

Now we use (4.1) with $\beta_{j}=0$ for $j \leqq m-1$ and $\varepsilon_{i} \psi_{m}(0)$ for $\beta_{m}$, and deduce as we did for (4.30),

$$
\begin{aligned}
& E_{\lambda_{0} \sigma_{i}}\left(x_{i}\right) \leqq \frac{1}{8^{l}} E_{8{ }^{l} \lambda_{0} \sigma_{l}}\left(x_{i}\right) \\
& +C_{3} \varepsilon_{i}^{2} \sum_{s=1}^{l} \frac{1}{8^{s}\left(8^{s} \lambda_{0}\right)^{n}} \int_{B_{8^{\prime} \lambda_{0}}(0) \backslash B_{8^{1}}{ }^{-1} \lambda_{0}(0)}\left\{\rho_{\infty}^{-2 \alpha} \sum_{j=1}^{m-1}\left|\psi_{j}^{i}-\psi_{j}\right|^{2}+\left|\psi_{j}\right|^{2}\right. \\
& \left.+\left|\psi_{m}-\psi_{m}(0)\right|^{2}+\left|\psi_{m}^{i}-\psi_{m}\right|^{2}\right\} d V \\
& \leqq \frac{1}{8^{l}} E_{8^{l} \lambda_{0} \lambda_{i}}\left(x_{i}\right) \\
& +\frac{C_{3} \varepsilon_{i}^{2}}{\lambda_{0}^{n}} \int_{B_{8^{l} \lambda_{0}}(0) \backslash B_{\lambda_{0}}(0)}\left(\rho_{\infty}^{-2 \alpha} \sum_{j=1}^{m-1}\left(\left|\psi_{j}^{i}-\psi_{j}\right|^{2}+\left|\psi_{m}^{i}-\psi_{m}\right|^{2}\right)\right) d V \\
& +C_{4} \varepsilon_{i}^{2}\left(8^{2 l} \lambda_{0}^{2}+(m-1) \sum_{s=1}^{l-1} \frac{1}{8^{s}} \sup _{B_{8^{s} \lambda_{0}}(0) \backslash B_{8^{s-1} \lambda_{0}}(0)} \rho_{\infty}^{\alpha}\right) \\
& \text { (use (4.29) and (4.40)) } \\
& \leqq \frac{1}{8^{l}} E_{8^{l} \lambda_{0} \sigma_{i}}\left(x_{i}\right)+\frac{C \frac{1}{8} \varepsilon_{i}^{2}}{\lambda_{0}^{n}} \int_{B_{8^{2} \lambda_{0}}(0) \backslash B_{\lambda_{0}}(0)}\left(\left|\psi_{m}^{i}-\psi_{m}\right|^{2}+\rho_{\infty}^{-2 \alpha} \sum_{j=1}^{m-1}\left|\psi_{j}^{i}-\psi_{j}\right|^{2}\right) \\
& +C_{4} \varepsilon_{i}^{2}\left(\lambda_{0}^{2}+(m-1) \sup _{B_{8^{2} \lambda_{0}}(0)} \rho_{\infty}^{\alpha}\right) \text {. }
\end{aligned}
$$


Note that both $C_{3}$ and $C_{4}$ here are uniform constants depending only on $n$, $C_{1 / 8}$ in (4.1) and $C$ in (4.40). Now we take $l_{0}$ to be $2\left(8 C_{4}(m-1)\right)^{1 / \alpha}$, and then choose $\lambda_{0}, l$ as before such that $1 / 4 l_{0} \leqq 2^{l} \lambda_{0} \leqq 1 / l_{0}$ and $\left(4 l_{0}\right)^{n-2} \leqq 8^{l-1}$. Since $B_{2 l} \lambda_{0}(0) \subset B_{1 / 2 l_{0}}(0)$ and $B_{1 / l_{0}(0)} \cap N_{\infty} \neq \varphi$, we have

$$
(m-1) C_{4} \sup _{B_{2^{\prime} \lambda_{0}}(0)} \rho_{\infty}^{\alpha} \leqq \frac{1}{8} .
$$

We further assume $C_{0} \lambda_{0} \leqq \frac{1}{8}$. Then by the strong convergence of $\psi_{j}^{i}$ to $\psi_{j}$ in $L^{2}$-norms, for $i$ sufficiently large, we have

$$
E_{\lambda_{0} \sigma_{i}}\left(x_{i}\right) \leqq \frac{1}{2} \varepsilon_{i}^{2}
$$

A contradiction! The proposition is proved. Note that $\lambda_{0}, \varepsilon_{0}$ are obviously independent of $x$ in $B_{\frac{1}{2}}\left(x_{0}\right)$.

Proof of Lemma 4.3. We will construct the barrier to obtain the decay estimate (4.39). First, we remark that it suffices to show (4.39) in a neighborhood of $N_{\infty} \cap B_{\frac{1}{2}}(0)$ in $B_{\frac{1}{2}}(0)$, since the coefficients of the linear equation (4.38) are smooth in any compact subset outside $N_{\infty}$.

Easy computations yield

$$
\begin{aligned}
&\left(\Delta-2\left(\frac{\alpha \nabla \rho_{\infty}}{\rho_{\infty}}+\nabla v\right) \cdot \nabla\right) \rho_{\infty}^{\gamma}=\gamma(\gamma-2 \alpha) \rho_{\infty}^{\gamma-2}-2 \alpha \gamma \nabla v \nabla \rho_{\infty} \cdot \rho_{\infty}^{\gamma-1} \\
&=\gamma \rho_{\infty}^{\gamma-2}\left\{(\gamma-2 \alpha)-2 \alpha \rho_{\infty} \nabla v \cdot \nabla \rho_{\infty}\right\}, \\
&\left(\Delta-2\left(\frac{\alpha \nabla \rho_{\infty}}{\rho_{\infty}}+\nabla v\right) \cdot \nabla\right) \rho_{\infty}^{\gamma-\delta} \tilde{r}_{\tilde{x}_{0}}(x)^{2}= \tilde{\gamma}_{\tilde{x}_{0}}(x)^{2}\left\{(\gamma-\delta)(\gamma-2 \alpha-\delta) \rho_{\infty}^{\gamma-\delta-2}\right. \\
&\left.-2 \alpha(\gamma-\delta) \rho_{\infty}^{\gamma-\delta-1} \nabla v \nabla \rho_{\infty}\right\} \\
&+2(n-2) \rho_{\infty}^{\gamma-\delta}-2 \alpha \rho_{\infty}^{\gamma-\delta} \nabla v \nabla \tilde{r}_{\bar{x}_{0}}(x)^{2} \\
&+2(\gamma-\delta) \rho_{\infty}^{\gamma-\delta-1} \nabla \rho_{\infty} \nabla \tilde{r}_{\tilde{x}_{0}}(x)^{2}
\end{aligned}
$$

where $\tilde{r}_{\bar{x}_{0}}(x)$ is the projection onto $N_{\infty}$ of the distance between $\bar{x}_{0}$ and $x$ for any fixed $\bar{x}_{0}$ in $B_{\frac{1}{2}}(0)$.

Therefore, for any given $\delta \leqq \gamma<2 \alpha$ and $0<\delta<1$, there is a neighborhood $U_{\gamma}$ of $N \cap B_{\frac{1}{2}}(0)$, depending only on $\varepsilon, \gamma, \beta$, such that

$$
\left(\Delta-2\left(\frac{\alpha \nabla \rho_{\infty}}{\rho_{\infty}}+\nabla v\right) \cdot \nabla\right)\left(\rho_{\infty}^{\gamma}+\rho_{\infty}^{\gamma-\delta} \tilde{r}_{\tilde{x}_{0}}(x)^{2}\right) \leqq-C^{\prime} \rho_{\infty}^{\gamma-2} \text { in } U_{\gamma} .
$$

Note that the assumption $|\nabla v| \leqq C \rho_{\infty}^{-\beta}(\beta<1)$ is used here. The function $\rho_{\infty}^{\gamma}+\rho_{\infty}^{\gamma-\delta} \tilde{r}_{x_{0}}(x)^{2} \geqq\left(\frac{2}{3}\right)^{2} \rho_{\infty}^{\gamma-\delta}$ for those $x$ with $\tilde{r}_{\bar{x}_{0}}(x) \geqq \frac{2}{3}$. So these $\rho_{\infty}^{\gamma}+\rho_{\infty}^{\gamma-\varepsilon} \tilde{r}_{\bar{x}_{0}}(x)^{2}$ can be served as upper barriers for $u$. Now fix $\tilde{\varepsilon}$ such that $l \tilde{\varepsilon}=2 \alpha-2 \varepsilon$ for some integer $l>0$. First we take $\delta=\gamma=\tilde{\varepsilon}$. Using maximum principle and comparing $u$ with $\rho_{\infty}^{\gamma}+\tilde{r}_{\bar{x}_{0}}(x)^{2}$ for any $\bar{x}_{0}$ in $N_{\infty} \cap B_{\frac{1}{2}}(0)$, we obtain

$$
|u(x)| \leqq C_{\alpha} \rho_{\infty}(x)^{\tilde{\varepsilon}} \quad \text { in } U_{\alpha} .
$$


Then we' repeat this argument successively with $\gamma=j \tilde{\varepsilon}, \delta=\tilde{\varepsilon}$ for $2 \leqq j \leqq l$ to conclude the proof of this lemma.

Remark. Proposition 4.1 can easily be modified in the axisymmetric case, where we need to construct an axisymmetric comparison map.

Now we are ready to give the main theorem of this section.

Theorem 4.1. There are two uniform constants $C$ and $\delta>0$ such that for any $x$ in $B_{\frac{1}{4}}\left(x_{0}\right), 0<\sigma \leqq \frac{1}{8}$,

$$
E_{\sigma}(x) \leqq C \sigma^{2 \delta}
$$

In particular, it implies that $\varphi_{m}$ is $\delta$-Hölder continuous (cf. [GT], Chap. 8).

Proof. Fix any $x$ in $B_{\frac{1}{4}}\left(x_{0}\right)$. Let $\varepsilon_{0}, \lambda_{0}$ be given in the last proposition. Then by Proposition 3.1, there is a $\sigma_{x}$ between $e^{-3 C / \varepsilon_{0}^{2}}$ and $\frac{1}{4}$, where $C$ is independent of $x$ in $B_{\frac{1}{2}}\left(\alpha_{0}\right)$ and $\left(\varphi_{1}, \ldots, \varphi_{m}\right)$, such that

$$
E_{\sigma_{x}}(x) \leqq \varepsilon_{0}^{2} .
$$

Combining this with the last proposition, we have

$$
E_{\lambda_{0}^{k} \sigma_{x}}(x) \leqq\left(\frac{1}{2}\right)^{k} E_{\sigma_{x}}(x)=\left(\frac{1}{2}\right)^{k} \varepsilon_{0}^{2} \quad \text { for } k \geqq 1 \text {. }
$$

Now for any $\sigma \leqq \frac{1}{8}$ and $\sigma \leqq \sigma_{x}$, choose $k$ such that $\lambda_{0}^{k+1} \sigma_{x}<\sigma \leqq \lambda_{0}^{k} \sigma_{x}$, then

$$
\begin{aligned}
E_{\sigma}(x) & \leqq \lambda_{0}^{-(n-2)} E_{\lambda_{0}^{k} \sigma_{x}}(x) \leqq \lambda_{0}^{-(n-2)} \varepsilon_{0}^{2}\left(\frac{1}{2}\right)^{k} \\
& \leqq \lambda_{0}^{-(n-2)} \varepsilon_{0}^{2}\left(\frac{1}{2}\right)^{-\frac{\log \sigma_{x}}{\log \lambda_{0}}} \sigma \frac{-\log 2}{\log \lambda_{0}} .
\end{aligned}
$$

Put $2 \delta=-\frac{\log 2}{\log \lambda_{0}}>0, C^{\prime}=\lambda_{0}^{-(n-2)} \varepsilon_{0}^{2}\left(\frac{1}{2}\right)^{-\frac{\log \sigma_{x}}{\log \lambda_{0}}}$, then

$$
E_{\sigma}(x) \leqq C^{\prime} \sigma^{2 \delta} \text { for } \sigma \leqq \min \left\{\frac{1}{8}, \sigma_{z}\right\} \text {. }
$$

On the other hand, $\sigma_{x}$ is uniformly bounded from below, therefore, the estimate (4.45) for $0 \leqq \sigma \leqq \frac{1}{8}$ follows from (4.46).

\section{Higher-order Estimates}

We will fix the solution $\left(\varphi_{1}, \ldots, \varphi_{m}\right)$ of (3.1) with $\left|\varphi_{m}\right| \leqq C$ in a geodesic ball $B_{1}\left(x_{0}\right)$ as in Sects. 3 and 4. Note that this center $x_{0}$ is in $N \subset M$ and $h_{m}$ is of the form (3.2) in the ball. In this section, we complete the proof of our main theorem stated in the introduction. We will always use $C$ to denote a uniform constant depending only on $\left(h_{1}, \ldots, h_{m}\right), N$, etc.

Lemma 5.1. There is an $\varepsilon_{0}>0$, independent of $\left(\varphi_{1}, \ldots, \varphi_{m}\right)$ and the point $x$ in $B_{\frac{1}{2}}\left(x_{0}\right)$, such that

$$
\left|\nabla \varphi_{m}\right| \leqq C \rho(x)^{-1+\varepsilon_{0}}, \quad x \text { in } B_{\frac{1}{2}}\left(x_{0}\right) \backslash N
$$


Proof. Given any point $x$ in $B_{\frac{1}{2}}(x) \backslash N$, put $2 \sigma=\rho(x)$. Then $\rho \geqq \sigma$ in $B_{\sigma}(x)$. Let $G_{z}(y)$ be the Green function in $B_{\sigma}(x)$ with Dirichlet boundary condition and singularity at $z$. Then one can easily check

$$
\begin{aligned}
& \int_{B_{\sigma}(x)}\left|\nabla G_{x}(y)\right|^{\beta} d V_{y} \leqq C_{\beta} \quad \text { for } \beta<\frac{n}{n-1}, \\
& \int_{\partial B_{\sigma}(x)}\left|\nabla \frac{\partial G_{x}(y)}{\partial n_{y}}\right| d V_{y} \leqq C,
\end{aligned}
$$

where $C_{\beta}$ depends only on $\beta$ and $n_{y}$ denotes the outward normal vector of $\partial B_{\sigma}(x)$.

Using the first equation in (3.1), we have

$$
\begin{aligned}
\varphi_{m}(z)-\varphi_{m}(x)= & \int_{\partial B_{\sigma}(x)}\left(\varphi_{m}(y)-\varphi_{m}(x)\right) \frac{\partial G_{z}(y)}{\partial n_{y}} d V_{y} \\
& -\int_{B_{\sigma}(x)} h_{m}^{-2} e^{-2 \varphi_{m}} \sum_{i=1}^{m-1}\left|\nabla \varphi_{i}\right|^{2}(y) G_{z}(y) d V_{y} .
\end{aligned}
$$

It follows

$$
\begin{aligned}
\left|\nabla \varphi_{m}(x)\right| \leqq & \int_{\partial B_{\sigma}(x)}\left|\varphi_{m}(y)-\varphi_{m}(x)\right| \nabla\left(\frac{\partial G_{x}}{\partial n_{y}}\right) \mid(y) d V_{y} \\
& +C_{1} \int_{B_{\sigma}(x)} \rho^{-2 \alpha} \sum_{i=1}^{m-1}\left|\nabla \varphi_{i}\right|^{2}(y)\left|\nabla G_{x}(y)\right| d V_{y}
\end{aligned}
$$

(Theorem 4.1 and (5.3)) $\leqq C_{2} \sigma^{\delta}+C_{1}\left(\int_{B_{\sigma}(x)} \rho^{-2 \alpha} \sum_{i=1}^{m-1}\left|\nabla \varphi_{i}\right|^{2}\left|\nabla G_{x}(y)\right| d V_{y}\right)$,

where $\delta>0$ is given in Theorem 4.1.

By Hölder inequality, we have

$$
\begin{aligned}
& \int_{B_{\sigma}(x)}\left(\rho^{-2 \alpha} \sum_{i=1}^{m-1}\left|\nabla \varphi_{i}\right|^{2}\right)\left|\nabla G_{x}(y)\right| d V_{y} \leqq\left(\int_{\beta_{-\sigma}(x)}\left(\rho^{-2 \alpha} \sum_{i=1}^{m-1}\left|\nabla \varphi_{i}\right|^{2}\right)^{\frac{\beta}{\beta-1}} d V_{y}\right)^{\frac{\beta-1}{\beta}} \\
& \times\left(\int_{B_{\sigma}(x)}\left|\nabla G_{x}(y)\right|^{\beta} d V_{y}\right)^{\frac{1}{\beta}}\left(\beta<\frac{n}{n-1}\right) \\
& \text { (Lemma 3.3) } \leqq C_{\beta}^{\frac{1}{\beta}} \cdot C_{\beta}^{\frac{\beta-1}{\beta}} \sigma^{-\frac{2}{\beta}}\left(\int_{B_{\sigma}(x)} \rho^{-2 \alpha} \sum_{i=1}^{m-1}\left|\nabla \varphi_{i}\right|^{2} d V_{y}\right)^{\frac{\beta-1}{\beta}}, \\
& \text { (Theorem 4.1) } \leqq C_{\beta}^{\frac{1}{\beta}} \cdot C_{4}^{\frac{\beta-1}{\beta}} \sigma^{-\frac{2}{\beta}+(n-2+2 \delta)^{\frac{\beta-1}{\beta}}} .
\end{aligned}
$$

Now choose $1<\beta<\frac{n}{n-1} \frac{n}{n-1}$ such that $(n-1) \beta-n+2 \delta(\beta-1)=\beta \varepsilon_{0}>0$ for some $\varepsilon_{0}>0$. Such a $\beta$ can be taken only dependent of $\delta$, so is $\varepsilon_{0}$. Then $-\frac{2}{\beta}+(n-2+\delta) \frac{\beta-1}{\beta} \geqq-1+\varepsilon_{0}$, and by (5.5), (5.6), we obtain

$$
\left|\nabla \varphi_{m}(x)\right| \leqq C_{1} \sigma^{\delta}+C_{5} \sigma^{-1+\varepsilon_{0}} \leqq C \rho(x)^{-1+\varepsilon_{0}} .
$$

The lemma is proved. 
Corollary 5.1. For any $\varepsilon>0, \varepsilon<\alpha$, there is a uniform constant $C_{\varepsilon}>0$ such that

$$
\left|\varphi_{j}(x)-h_{j}(\pi(x))\right| \leqq C_{\varepsilon} \rho(x)^{2 \alpha-2 \varepsilon}, \quad x \text { in } B_{\frac{1}{2}}\left(x_{0}\right)
$$

where $j=1,2, \ldots, m-1$ and $\pi$ is the projection from $B_{1}\left(x_{0}\right)$ into $N$ as given in Corollary 3.1.

Proof. In case $\alpha \geqq 1, h_{j} \equiv$ const. in $B_{1}\left(x_{0}\right) \cap N$. Then (5.8) follows from the previous lemma and Lemma 4.3. In case $\alpha<1$, we may extend $h_{j}$ such that $\nabla h_{j} \nabla \rho \leqq C \rho$ near $N$. Then we apply Lemma 4.3 to $\varphi_{j}-h_{j}$ and obtain the estimate (5.8). Note that $\rho_{\infty}=\rho$ and $N_{\infty}=N$ in the application of Lemma 4.3.

In particular, the solution $\left(\varphi_{1}, \ldots, \varphi_{m}\right)$ is $\delta$-Hölder continuous and its $\delta$-Hölder norm is uniformly bounded, where $\delta=\delta(\alpha)$ depends only on $\alpha$. However, in case $\alpha>1$, we can have more estimates on the second derivatives of this solution.

Corollary 5.2. Suppose that $\alpha>1$ and $h_{m}$ is of $C^{1, \beta}$, where $l \in Z_{+}$and $0<\beta<1$. Then for any $\varepsilon>0, \varepsilon<2 \alpha$, there is a uniform constant $C_{\varepsilon}>0$ such that

$$
\max _{1 \leqq j \leqq m}\left\{\left\|\varphi_{j}\right\|_{C_{\alpha}^{k_{\alpha}, \lambda\left(B_{\frac{1}{2}}\left(x_{0}\right)\right)}}\right\} \leqq C_{\varepsilon}
$$

where $k_{\alpha}=\min \{[2 \alpha-2 \varepsilon], l+1\}$, and $\lambda_{\alpha}=\min \left\{2 \alpha-2 \varepsilon-k_{\alpha}, \beta\right\}$.

Proof. Note that $h_{j} \equiv$ const. for $j=1,2, \ldots, m-1$ in case $\alpha>1$. By Corollary 5.1 , it suffices to bound on the $\left(k_{\alpha}, \lambda_{\alpha}\right)$-Hölder norm of $\varphi_{m}$ in $B_{\frac{1}{2}}\left(x_{0}\right)$. In the first equation of (3.1), $\varphi_{m}$ is Hölder continuous and $h_{m}^{-2} \sum_{j=1}^{m-1}\left|\nabla \varphi_{j}\right|^{2}$ is $([2 \alpha-2-2 \varepsilon]$, $2 \alpha-2-2 \varepsilon-[2 \alpha-2 \varepsilon-2])$-Hölder continuous by Corollary 5.1. Therefore the bound on the $\left(k_{\alpha}, \lambda_{\alpha}\right)$-Hölder norm of $\varphi_{m}$ follows from the standard Schauder estimate (cf. [GT]).

Theorem 1.1 follows from Corollary 5.1 and 5.2.

In the following, we will prove that the solution $\left(\varphi_{1}, \ldots, \varphi_{m}\right)$ has more regularity along the tangential directions of $N$ in $B_{\frac{1}{2}}\left(x_{0}\right)$, precisely, by a local diffeomorphism, we may assume that $B_{1}\left(x_{0}\right)$ is an open neighborhood of the origin in $R^{n}$ with $N \cap B_{1}\left(x_{0}\right)$ being in the subspace $R^{n-2} \subset R^{n}$, let $\left(x_{1}, \ldots, x_{n}\right)$ be the Euclidean coordinates, then $\frac{\partial^{l} \varphi_{j}}{\partial x_{1}^{l_{1}} \ldots \partial x_{n-2}^{l_{n-2}}}\left(l_{1}+\cdots+l_{n-2}=l\right)$ have the same estimate as (5.9) in the half-ball $B_{\frac{1}{2}}\left(x_{0}\right)$ as long as $h_{m}, N$ and the metric $d s^{2}$ are of $C^{l+2}$ in $B_{1}\left(x_{0}\right)$. To avoid the complexity from the presence of curvature of $N$ and $d s^{2}$, we simply consider the case where $M=R^{n} . N=R^{n-2} \subset R^{n}$ and $d s^{2}$ is the flat metric. The proof for general cases is identical. Furthermore, we assume that $\log h_{m}$ is harmonic outside $N$. By adding a $C^{\infty}$-function to $\varphi_{m}$, we may take $h_{m}$ to be $\rho^{\alpha}$, where $\alpha>1$ and $\rho\left(x_{1}, \ldots, x_{n}\right)=\sqrt{x_{n-1}^{2}+x_{n}^{2}}$. The equations in (3.1) become

$$
\begin{gathered}
\Delta \varphi_{m}=\rho^{-2 \alpha} e^{-2 \varphi_{m}} \sum_{i=1}^{m-1}\left|\nabla \varphi_{j}\right|^{2} \\
\Delta \varphi_{j}=2 \nabla \varphi_{j}\left(\frac{\alpha \nabla \rho}{\rho}+\nabla \varphi_{m}\right), \quad j=1,2, \ldots, m-1 .
\end{gathered}
$$


Lemma 5.2. For any integer $l \geqq 1$, and nonnegative integers $l_{1}, \ldots, l_{n-2}$ with $l=l_{1}+\cdots+l_{n-2}$, we have

$$
\begin{aligned}
\Delta\left(\frac{\partial^{l} \varphi_{m}}{\partial x_{1}^{l_{1}} \ldots \partial x_{n-2}^{l_{n-2}}}\right)= & -2 \rho^{-2 \alpha} e^{-2 \varphi_{m}} \sum_{j=1}^{m-1}\left|\nabla \varphi_{j}\right|^{2} \cdot \frac{\partial^{l} \varphi_{m}}{\partial x_{1}^{l_{1}} \ldots \partial x_{n-2}^{l_{n-2}}} \\
& +2 \rho^{-2 \alpha} e^{-2 \varphi_{m}} \sum_{j=1}^{m-1} \nabla \varphi_{j} \nabla\left(\frac{\partial^{l} \varphi_{j}}{\partial x_{1}^{l_{1}} \ldots \partial x_{n-2}^{l_{n-2}}}\right) \\
& +P_{l_{1}, \ldots, l_{n-2}}^{m} \rho^{-2 \alpha} e^{-2 \varphi_{m}} \\
\Delta\left(\frac{\partial^{l} \varphi_{j}}{\partial x_{1}^{l_{1}} \ldots \partial x_{n-2}^{l_{n-2}}}\right)= & 2 \nabla\left(\frac{\partial^{l} \varphi_{j}}{\partial x_{1}^{l_{1}} \ldots \partial x_{n-2}^{l_{n-2}}}\right) \cdot\left(\frac{\alpha \nabla}{\rho}+\nabla \varphi_{m}\right) \\
+ & 2 \nabla \varphi_{j} \nabla\left(\frac{\partial^{l} \varphi_{m}}{\partial x_{1}^{l_{1}} \ldots \partial x_{n-2}^{l_{n-2}}}\right) \\
& +P_{l_{1}, \ldots, l_{n-2}}^{j}, j=1,2, \ldots, m-1
\end{aligned}
$$

where $P_{l_{1}, \ldots, l_{n-2}}^{j}, P_{l_{1}, \ldots, l_{n-2}}^{m}$ are polynomials of degree at most $l+2$ in $\frac{\partial^{k} \varphi_{m}}{\partial x_{1}^{k_{1}} \ldots \partial x_{n-2}^{k_{n-2}}}$ and $\nabla\left(\frac{\partial^{k} \varphi_{m}}{\partial x_{1}^{k_{1}} \ldots \partial x_{n-2}^{k_{n-2}}}\right)$, where $0 \leqq k \leqq l-1, k_{1}+\cdots+k_{n-2}=k$. Furthermore, $P_{l_{1}, \ldots, l_{n-2}}^{m}$ is at least quadratic on the derivatives of $\varphi_{j}(j=1,2, \ldots, m-1)$.

Proof. These equations follow from the direct computations, (5.10) and (5.11).

Theorem 5.1. Let $N=R^{n-2} \subset R^{n}=M$ be the subspace, $h_{1}, \ldots, h_{m}$ be $C^{\infty}$-functions with $h_{m}=\rho^{\alpha}$ in $B_{1}(0)$. Then for any $(n-2)$-tuple of nonnegative integers $\left(l_{1}, \ldots, l_{n-2}\right)$ and $\varepsilon>0$, there is a constant $C_{\varepsilon, l}$, depending only on $\varepsilon$ and $l=l_{1}+\cdots+l_{n-2}$, such that

$$
\max _{1 \leqq j \leqq m}\left\{\left\|\frac{\partial^{l} \varphi_{j}}{\partial z_{1}^{l_{1}} \ldots \partial z_{n-2}^{l_{n-2}}}\right\|_{C^{k_{\alpha} \cdot \lambda}\left(B_{\frac{1}{2}}(0)\right)}\right\} \leqq C_{\varepsilon, l},
$$

where $k_{\alpha}=[2 \alpha-2 \varepsilon], \lambda_{\alpha}=2 \alpha-2 \varepsilon-k_{\alpha}$ as in Corollary 5.2.

Proof. We will prove (5.14) by induction on $l=\sum_{i=1}^{n-2} l_{i}$ for fixed small $\varepsilon>0$. In case $l=0,(5.14)$ is just (5.9). Now we suppose that (5.14) is true for all $\left(l_{1}^{\prime}, \ldots, l_{n-2}^{\prime}\right)$ with $l_{1}^{\prime}, \ldots, l_{n-2}^{\prime}<l$. By induction, we may further assume that on $B_{\frac{3}{4}}(0)$,

$$
\left|\frac{\partial^{k} \varphi_{j}}{\partial x_{1}^{k_{1}} \ldots \partial x_{n-2}^{k_{2}}}\right|(x) \leqq C_{k, \varepsilon} \rho(x)^{2 \alpha-2 \varepsilon}
$$

and

$$
\left|\nabla\left(\frac{\partial^{k} \varphi_{j}}{\partial x_{1}^{k_{1}} \ldots \partial x_{n-2}^{k^{2}}}\right)\right|(x) \leqq C_{k, \varepsilon} \rho(x)^{2 \alpha-2 \varepsilon-1},
$$

where $0 \leqq k \leqq l-1,1 \leqq j \leqq m-1$ and $C_{k, \varepsilon}$ are constants independent of the solution. 
Then by Lemma 5.2, in Eqs. (5.12) and (5.13), for some uniform constant $C$,

$$
\begin{gathered}
\left|P_{l_{1}, \ldots, l_{n-2}}^{m}(x)\right| \leqq C \rho^{4 \alpha-4 \varepsilon-2}(x) \quad x \text { in } B_{\frac{1}{2}}(0), \\
\left|P_{l_{1}, \ldots, l_{n-2}}^{j}(x)\right| \leqq C \rho^{2 \alpha-2 \varepsilon-1}(x), \quad j=1,2, \ldots, m-1 .
\end{gathered}
$$

On the other hand, by induction

$$
\begin{aligned}
\left|\nabla\left(\frac{\partial^{l} \varphi_{m}}{\partial x_{1}^{l_{1}} \ldots \partial x_{n-2}^{l_{n-2}}}\right)\right|(x) & \leqq \max \left\|\frac{\partial^{l-1} \varphi_{m}}{\partial x_{1}^{k_{1}} \ldots \partial x_{n-2}^{k_{n-2}}}\right\|_{C^{2}\left(B_{\frac{1}{2}}(0)\right) \mid k_{1}+\cdots+k_{n-2}=l-1} \\
& \leqq C_{\varepsilon, l-1} .
\end{aligned}
$$

Therefore

$$
\left|2 \nabla \varphi_{j} \nabla\left(\frac{\partial^{l} \varphi_{m}}{\partial x_{1}^{l_{1}} \ldots \partial x_{n-2}^{l_{n-2}}}\right)+P_{l_{1}, \ldots, l_{n-2}}^{j}\right|(x) \leqq C \rho^{2 \alpha-2 \varepsilon-1} \leqq C \rho^{2 \alpha-2} .
$$

Now we can apply Lemma 4.3 to (5.13) with $u=\frac{\partial^{l} \varphi_{j}}{\partial x_{1}^{l_{1}} \ldots \partial x_{n-2}^{l_{n-2}}}$ for $j=1,2, \ldots, m-1$ to conclude

$$
\left|\frac{\partial^{l} \varphi_{j}}{\partial x_{1}^{l_{1}} \ldots \partial x_{n-2}^{l_{n-2}(x)}}\right| \leqq C \rho(x)^{2 \alpha-2 \varepsilon}, \quad x \text { in } B_{\frac{1}{2}}(0)
$$

Note that the estimate for $\frac{\partial^{l} \varphi_{j}}{\partial x_{1}^{l_{1}} \ldots \partial x_{n-2}^{l_{n-2}(x)}}$ corresponding to (5.5) and (5.16) can be easily derived from (5.19). Therefore, (5.14) follows from (5.19), (5.12), (5.13) and the standard Schauder estimates (cf. [GT], Chap. 6).

Theorem 5.1' (The version of Theorem 5.1 in general case). Assume that $M$ is a $C^{\infty}$-manifold, $N$ is a $C^{\infty}$-submanifold and $\left(h_{1}, \ldots, h_{m}\right)$ are $C^{\infty}$-smooth. Let $B_{1}\left(x_{0}\right)$ be the geodesic ball with $x_{0}$ in $N$ in which $h_{m}$ is of form (3.2). Then for any $\varepsilon>0$, any vector fields $T_{1}, \ldots, T_{l}$ in $B_{1}\left(x_{0}\right)$ which are tangential to $N$ along $B_{1}\left(x_{0}\right) \cap N$, there is a constant $C_{\varepsilon}=C_{\varepsilon}\left(T_{1}, \ldots, T_{l}\right)$, independent of the solution $\left(\varphi_{1}, \ldots, \varphi_{m}\right)$, such that

$$
\sup _{1 \leqq j \leqq m}\left\|T_{1} \ldots T_{l} \varphi_{j}\right\|_{C^{k_{\alpha}, \lambda_{\alpha}\left(B_{\frac{1}{2}}\left(x_{0}\right)\right)}} \leqslant C_{\varepsilon, l},
$$

where $k_{\alpha}, \lambda_{\alpha}$ are given as in Theorem 5.1.

The proof of it is identical to that of Theorem 5.1 except for some possible complexity due to the bending of $M$ and $N$.

\section{An Application}

In this section, we apply the regularity theorem in Sect. 5 for harmonic maps into $H^{2}$ to the uniqueness problem of axially symmetric, asymptotically flat, stationary spacetimes (cf. [Ca, We] for the definition) in general relativity.

The Einstein vacuum field equations of general relativity are

$$
\operatorname{Ric}(g)=0 \text {, }
$$


where $(M, g)$ is a 4-dimensional Lorentzian manifold and $\operatorname{Ric}(g)$ denotes its Ricci curvature. In view of the great difficulties involved in the study of these equations, one is led to consider special cases with symmetry. The solutions of Schwarzschild are found in 1916, the first explicit ones parameterized by the mass. They are all static and spherically symmetric. The Kerr family of solutions to (6.1), discovered in 1963, has two parameters, the mass and the aungular momentum, and is both stationary and axially symmetric. In the 70's Robinson shows that the Kerr solutions are unique among all asymptotically flat axially symmetric stationary spacetimes that have a connected event horizon. His proof is based on some results of Ernst [Er] and Carter [Ca]. They reduce the Einstein vacuum equation in the asymptotically flat, axially symmetric stationary case to an axially symmetric harmonic map from 3-dimensional euclidean space into a hyperbolic plane $H^{2}$. Then the uniqueness of Kerr solutions is the same as that of the harmonic map into $H^{2}$. Robinson affirmed the later by using the convexity of the distance function on $H^{2}$.

After this striking result of Robinson, there is a "little" problem left, i.e., is it possible to drop the extra assumption on the connectedness of the event horizon. Following a suggestion of D. Christodoulou, G. Weinstein considered this problem in his thesis [We1]. In order to describe his basic result, we need to first introduce some notations.

Let $(\rho, \varphi, z)$ be the cylindrical coordinates for $R^{3}$ and $A$ be the $z$-axis. Choose parameters $\left\{a_{i}, b_{i}, c_{i}\right\}_{1 \leqq i \leqq L}$ for any fixed positive integer $L$ satisfying:

$$
-\infty<a_{1}<b_{1}<a_{2}<\cdots<b_{L-1}<a_{L}<b_{L}=0 \text {. }
$$

Define $\Gamma=A \backslash\left\{(0, \varphi, z) \mid a_{i}<z<b_{i}\right.$ for some $\left.i\right\}$. Then the distance function $d((\rho, \varphi, z), \Gamma)$ is Lipschitz in $R^{3}$ and the Laplacian of its logarithm is bounded except at those boundary points. By Fourier transformation, one can find a smooth function $u$ outside $A \backslash \Gamma$ such that $h=2 \log d(\cdot, \Gamma)+u$ is harmonic outside $\Gamma$ and $h$ is asymptotic to $2 \log d(\cdot, \Gamma)$ at the interior points of $\Gamma$. In fact, such a $h$ can be explicitly obtained by superposition of Schwarzschild metrics. Namely, we can write

$$
\begin{aligned}
& h=2 \log \rho+\sum_{i=1}^{L} u_{i}, \\
& u_{i}=-\log \left(1-\frac{b_{i}-a_{i}}{r_{i}}\right),
\end{aligned}
$$

where $\left(r_{i}, \theta_{i}\right)$ are given by transformations

$$
\begin{aligned}
\rho & =r_{i}\left(1-\frac{b_{i}-a_{i}}{r_{i}}\right)^{\frac{1}{2}} \sin \theta_{i}, \\
z-\frac{b_{i}+a_{i}}{2} & =\left(r_{i}-\frac{b_{i}-a_{i}}{2}\right) \cos \theta_{i}
\end{aligned}
$$

(cf. $[\mathrm{We1}])$.

Let $(X, Y)$ be a harmonic map from $R^{3} \backslash A$ into the upper half plane with standard hyperbolic metric, satisfying. 
$X$ is smooth across $\Gamma$ away from its boundary points and,

$$
\begin{aligned}
& X(0, \varphi, z)=c_{i} \quad \text { for } b_{i}<z<a_{i+1}, \quad i=1,2, \ldots, L-1, \\
& X(0, \varphi, z)=c_{L} \quad \text { for } z>b_{L}, \\
& X(0, \varphi, z)=-c_{L} \text { for } z<a_{1},
\end{aligned}
$$

both $X$ and $Y$ are axially symmetric, i.e., they are independent of $\varphi$. (6.7)

$Y$ is of the form $e^{h+y}$ and $y$ is smooth across $\Gamma$ away from

its boundary points.

Both $X$ and $y$ are of order $O\left(\frac{1}{\sqrt{\rho^{2}+z^{2}}}\right)$ as $(\rho, \varphi, z)$ near infinity .

By the harmonicity of $(X, Y)$ and (6.3)-(6.5), one can easily check that the differential 1-form $\Psi$ is closed and smooth in $R^{3} \backslash(\overline{A \backslash T})$, where $\overline{A \backslash \Gamma}$ denotes the closure of $A \backslash \Gamma$ and

$$
\begin{aligned}
\Psi= & \frac{1}{4} \rho Y^{-2}\left\{\left(\frac{\partial Y}{\partial \rho}\right)^{2}+\left(\frac{\partial X}{\partial \rho}\right)^{2}-\left(\frac{\partial Y}{\partial z}\right)^{2}-\left(\frac{\partial X}{\partial z}\right)^{2}\right\} d \rho \\
& +\frac{1}{2} \rho X^{-2}\left\{\left(\frac{\partial Y}{\partial \rho}\right)\left(\frac{\partial Y}{\partial z}\right)+\left(\frac{\partial X}{\partial \rho}\right)\left(\frac{\partial X}{\partial z}\right)\right\} d z-d \log \rho .
\end{aligned}
$$

Therefore, there is a smooth function $\omega$ in $R^{3} \backslash(\overline{A \backslash \Gamma})$ satisfying $d \omega=\Psi$. Such an $\omega$ is unique up to constants, so we may normalize $(\omega-u-y)(0, \varphi, 1)=0$. The following proposition is essentially due to Ernst [Er] and Carter [Ca], but the form of it presented here is formulated in [We1] with some changes on the notations.

Proposition 6.1. The asymptotically flat axially symmetric stationary solutions of EVE (6.1) with $n$ connected components of even horizon are equivalent to those harmonic maps described as above with one extra condition

$$
\beta \equiv 0 \quad \text { on } \Gamma \backslash \partial \Gamma,
$$

where $\beta$ is defined to be $\omega-u-y$ in $R^{3} \backslash(\overline{A \backslash \Gamma})$.

Note that our EVE solutions always have a nondegenerate event horizon in the sense in [Ca]. Also, the condition (6.11) is nothing else but an interpretation of the regularity of the EVE solutions along the axis away from event horizon.

The parameters $\left\{a_{i}, b_{i}, c_{i}\right\}_{1 \leqq i \leqq L}$ have the following physical interpretations. The differences $a_{i+1}-b_{i}(1 \leqq i \leqq L-1)$ are the distances between two adjacent components of the event horizon, the length $b_{i}-a_{i}$ of the interval $\left[a_{i}, b_{i}\right]$ can be regarded as the mass of the $i^{\text {th }}$-component of an event horizon. Finally, the angular momentum $J_{i}$ of the $i^{\text {th }}$-component is $\frac{1}{8}\left(c_{i}-c_{i-1}\right)$ for $i \geqq 2$ and $\frac{1}{8}\left(c_{1}+c_{L}\right)$ for $i=1$. In particular, the total angular momentum is $\frac{1}{4} c_{L}$. We refer the readers to Sect. 6 in [We1] for details. The solutions of $E V E$ (6.1) without rotation, i.e., all $c_{i}$ vanishes, were discussed a long time ago by Bach and Weyl [BW]. The corresponding harmonic maps are of form $\left(0, \rho^{2} e_{i=1}^{L} u_{i}\right)$, where $u_{i}$ are given by (6.3)-(6.5). Let us first compute $\beta$ on the bounded components of $\Gamma$ for these particular nonrotational solutions. 
Lemma 6.1. Let $\left(0, \rho^{2} e_{i=1}^{L} u_{i}\right)$ be the harmonic map corresponding to one solution of Bach and Weyl, i.e. all $c_{i}$ vanish. Then for $z \in\left(b_{i}, a_{i+1}\right), i=1,2, \ldots, L-1$,

$$
\begin{aligned}
\beta(0, \varphi, z)= & -\sum_{k=i+1}^{L} \sum_{j=1}^{i} \log \frac{\left(b_{k}-b_{j}\right)\left(a_{k}-a_{j}\right)}{\left(b_{k}-a_{j}\right)\left(a_{k}-b_{j}\right)} \\
& <0 \\
\beta(0, \varphi, z)= & 0 \text { for either } z>b_{L} \text { or } z<a_{1} .
\end{aligned}
$$

Proof. Let $C_{\varepsilon}^{i}$ be a path in the $(\rho, z)$-plane defined by the equation $r_{i}=2 m_{i}+\varepsilon$, where $r_{i}$ is defined in (6.4) and (6.5), $m_{i}$ is equal $\frac{b_{i}-a_{i}}{2}$. Put $d_{i}=\frac{a_{i}+b_{i}}{2}$. Then by computations, we find

$$
\begin{aligned}
\frac{\partial u_{i}}{\partial \rho}= & \frac{-2 m_{i}}{r_{i}^{\frac{1}{2}}\left(r_{i}-2 m_{i}\right)^{\frac{1}{2}}}\left(r_{i}-m_{i}\right) \sin \theta_{i}\left\{\left(r_{i}-m_{i}\right)^{2} \sin ^{2} \theta_{i}\right. \\
& \left.+r_{i}\left(r_{i}-2 m_{i}\right) \cos ^{2} \theta_{i}\right\}^{-1} \\
\frac{\partial u_{i}}{\partial z}= & -2 \cos \theta_{i}\left\{\left(r_{i}-m_{i}\right)^{2} \sin ^{2} \theta_{i}\right. \\
& \left.+r_{i}\left(r_{i}-2 m_{i}\right) \cos ^{2} \theta_{i}\right\}^{-1}
\end{aligned}
$$

where $i=1,2, \ldots, L$.

In particular, it implies that both $\frac{\partial u_{i}}{\partial z}$ and $\frac{\partial u_{i}}{\partial \rho}$ are smooth in the region $\left\{r_{i}>2 m_{i}\right\}$. Since $\left(0, \rho^{2}, e^{u_{i}}\right)$ corresponds to the Schwarzschild solution, we have

$$
\frac{1}{4} \int_{C_{t}^{i}}\left\{\rho\left(\left(\frac{\partial u_{i}}{\partial \rho}\right)^{i}-\left(\frac{\partial u_{i}}{\partial z}\right)^{i}\right) d \rho+2 \rho \frac{\partial u_{i}}{\partial \rho} \frac{\partial u_{i}}{\partial z} d z\right\}=0
$$

Therefore, using (6.13), (6.14) and (6.15), for $z_{2}$ in $\left(b_{i}, a_{i+1}\right)$ and $z_{1}$ in $\left(b_{i-1}, a_{i}\right)$, we have

$$
\begin{aligned}
\beta\left(0, \varphi, z_{2}\right)-\beta\left(0, \varphi, z_{1}\right)= & \lim _{\varepsilon \rightarrow 0+C_{\varepsilon}^{i}} d \beta \\
= & \frac{1}{4} \lim _{\varepsilon \rightarrow 0+} \int_{C_{\varepsilon}^{i}}\left\{\rho\left(\left(\frac{\partial u}{\partial \rho}\right)^{2}-\left(\frac{\partial u}{\partial z}\right)^{2}\right) d \rho\right. \\
& \left.+2 \rho \frac{\partial u}{\partial \rho} \frac{\partial u}{\partial z} d z\right\} . \\
= & \frac{1}{2} \lim _{\varepsilon \rightarrow 0+} \int_{C_{\varepsilon}^{i}}\left(\sum_{j \neq i} d u_{j}\right) \cdot \frac{\partial u_{i}}{\partial \rho} \\
= & -\sum_{j \neq i} u_{j}\left(b_{i}\right)-u_{j}\left(a_{i}\right) \\
= & \sum_{j>i} \log \frac{\left(a_{j}-b_{i}\right)\left(b_{j}-a_{i}\right)}{\left(b_{j}-b_{i}\right)\left(a_{j}-a_{i}\right)} \\
& +\sum_{j<i} \log \frac{\left(b_{i}-b_{j}\right)\left(a_{i}-a_{j}\right)}{\left(b_{i}-a_{j}\right)\left(a_{i}-b_{j}\right)} .
\end{aligned}
$$

Now our normalization says $\beta(0, \varphi, 1)=0$, so (6.12) follows from (6.16)! 
In particular, any nonrotational asymptotically flat axially symmetric stationary of (6.1) is not regular on the bounded components of the axis away from event horizon.

In [We1], G. Weinstein considered the construction of harmonic maps from $R^{3} \backslash A$ into $H^{2}$ of form $\left(X, e^{h+y}\right)$, where $h$ is equal to $2 \log \rho+\sum_{i=1}^{L} u_{i}$ with $u_{i}$ given in (6.3)-(6.5). His idea is to minimize the following functional

$$
H(X, y)=\int_{R^{3} \backslash A}\left(|\nabla y|^{2}+e^{-2 h-2 y}|\nabla X|^{2}\right) d V
$$

in the space $H_{1, h} \times H_{1}$ (cf. Sect. 2 or Sect. 6 in [We1]).

Proposition 6.2. For any set of numbers $\left\{a_{i}, b_{i}, c_{i}\right\}_{1 \leqq i \leqq L}$ satisfying (6.2), there is a unique axially symmetric harmonic map of form $\left(X, e^{h+y}\right)$ from $R^{3} \backslash A$ into $H^{2}$ satisfying:

and

$$
\int_{R^{3}}\left(|\nabla y|^{2}+e^{-2 h-2 y}|\nabla X|^{2}\right) d V \leqq C
$$

$$
\sup _{R^{3} \backslash A}|y| \leqq C,
$$

where $C$ is a constant depending only on $b_{L}-a_{1}$ and $\max _{1 \leqq i \leqq L}\left|c_{i}\right|$.

Proof. This is due to Weinstein (cf. in [We1]).

The following is just a special case of Theorem 1.1.

Theorem 6.1. For any set of numbers $\left\{a_{i}, b_{i}, c_{i}\right\}_{1 \leqq i \leqq L}$ satisfying (6.2), there is a unique harmonic map from $R^{3} \backslash A$ into $H^{2}$ such that (6.8)-(6.9) hold. Moreover, the $C^{2, \frac{1}{2}}$-norms of $(X, y)$ in any compact subset $K \subset \subset R^{3} \backslash A$ are uniformly bounded by a constant depending only on $K$ and $C$ in (6.18) and (6.19).

Theorem 6.2. Given any two numbers $\lambda_{1}, \lambda_{2}>0$, there is an $\varepsilon=\varepsilon\left(\lambda_{1}, \lambda_{2}\right)>0$ such that for any set of $\left\{a_{i}, b_{i}, c_{i}\right\}_{1 \leqq i \leqq L}$ satisfying $\max _{1 \leqq i \leqq L}\left|c_{i}\right| \leqq \varepsilon, b_{L}-a_{1} \leqq \lambda_{2}$ and

$$
\min \left\{\inf _{1 \leqq i \leqq L}\left(b_{i}-a_{i}\right), \inf _{1 \leqq i \leqq L-1}\left(a_{i+1}-b_{i}\right)\right\} \geqq \lambda_{1},
$$

the function $\beta$ defined in Proposition 6.1 is negative in each bounded component of $\Gamma$. Equivalently, there is no regular asymptotically flat axially symmetric stationary solution of EVE (6.1) such that its event horizon has $L$ connected components disjoint from each other in the distance at least $\lambda_{1}$ and at most $\lambda_{2}$, and each component has mass $\geqq \lambda_{1}$ and angular momentum less than $\varepsilon$.

Proof. We observe that the $C^{2, \frac{1}{2}}$-estimates for $(X, y)$ in Sect. 5 are uniform if $(6.18)$ and (6.19) hold (cf. Theorem 6.1). On the other hand, the harmonic map $(X, Y)$ does satisfy (6.18)-(6.19) for a constant $C$ depending only on $\lambda_{1}, \lambda_{2}$ under our assumption on $a_{i}, b_{i}, c_{i}(1 \leqq i \leqq L)$ (cf. Sect. 2). Therefore, this theorem follows from a continuity argument and Lemma 6.1.

Theorem 6.3. Let $\left(X_{\alpha}, Y_{\alpha}\right)$ be a sequence of harmonic maps from $R^{3} \backslash A$ ino $H^{2}$ satisfying (6.6)-(6.9) for $\left\{a_{\alpha i}, b_{\alpha i}, c_{\alpha i}\right\}_{1 \leqq i \leqq L}$. Suppose that

(1) $\sup _{\alpha, i}\left\{\left|b_{\alpha i}-a_{\alpha i}\right|,\left|c_{\alpha i}\right|\right\} \leqq C$,

(2) $\exists i_{0}$, s.t. $\lim _{\alpha \rightarrow \infty}\left(a_{\alpha i_{0}+1}-b_{\alpha i_{0}}\right)=\infty$ and $\sup _{\alpha, i \neq i_{0}}\left(\left|a_{\alpha i+1}-b_{\alpha i}\right|\right) \leqq C$,

where $C$ is a uniform constant. Then $\left(X_{\alpha}, Y_{\alpha}\right)$ converges to the union of two harmonic maps from $R^{3} \backslash A$ into $H^{2}$ satisfying (6.6)-(6.9) for two sets of numbers 


$$
\begin{array}{rlrl}
\left\{a_{\infty i}, b_{\infty i}, c_{\infty i}\right\}_{i \leqq i \leqq i_{0}} \text { and }\left\{a_{\infty i}, b_{\infty i}, c_{\infty i}\right\}_{i_{0} \leqq i \leqq L}, \text { respectively, where } & \\
a_{\infty i} & =\lim _{\alpha \rightarrow \infty}\left(a_{\alpha i}-b_{\alpha i_{0}}\right), & b_{\infty i}=\lim _{\alpha \rightarrow \infty}\left(b_{\alpha i}-b_{\alpha i_{0}}\right) \quad \text { for } i \leqq i_{0}, \\
a_{\infty i} & =\lim _{\alpha \rightarrow \infty}\left(a_{\alpha i}-a_{\alpha i_{0}+1}\right), & b_{\infty i}=\lim _{\alpha \rightarrow \infty}\left(b_{\alpha i}-b_{\alpha i_{0}+1}\right) & \text { for } i>i_{0}, \\
c_{\infty i} & =\lim _{\alpha \rightarrow \infty} c_{\alpha i} . &
\end{array}
$$

We omit the proof. It is simply a corollary of the results in Sect. 2, the regularity theorem in Sect. 5 and some standard arguments.

In particular, this last theorem implies that the solution of $E V E$ (6.1) with two black holes constructed in Sect. 8 of [We] converges to the union of two Kerr's solutions with opposite total angular momentum as the distance of two black holes approaches to infinity.

\section{References}

[Ca] Carter, B.: Black hole equilibrium states, in Black holes. Dewitt, C. DeWitt, B.S. (eds.) New York: Gordon and Breach Science Publishers 1973

[ES] Eells, J., Sampson, J.H.: Harmonic mappings of Riemannian manifolds. Am. J. Math. 86, 109-160 (1964)

[Er] Ernst, F.J.: New formulation of the gravitational field problem. Phys. Rev. Lett. 167, $1175-1178$ (1968)

[Gi] Giaquinta, M., Giusti, S.: On regularity of the minima of variational integrals. Acta Math. 148, 31-40 (1982)

[GT] Gilbarg, D., Trudinger: Elliptic partial differential equations of second order. Berlin, Heidelberg, New York: Springer, $2^{\text {nd }}$ edition, 1983

[Ha] Hamilton, R.S.: Harmonic maps of manifolds with boundary. Lecture Notes vol. 471. Berlin, Heidelberg, New York: Springer 1975

[HL] Hardt, R., Lin, F.H.: Mappings minimizing the $L^{p}$ norm of the gradient. Commun. Pure Appl. Math. XL, 555-588 (1987)

[HW] Hildebrandt, S., Widman, K.-O.: On the Hölder continuity of weak solutions of quasilinear elliptic systems of second order. Ann. Scuola Norm. Sup. Pisa (IV) 4, 145-178 (1977)

[LT1] Yanyan Li, Gang Tian: Harmonic maps with prescribed singularities. To appear in the Proceedings of 1989 Summer Institute, UCLA

[LT2] Yanyan Li: Nonexistence of axially symmetric, stationary solution of Einstein Vacuum Equation with disconnected symmetric event horizon. Manus. Math. 78, 83-89 (1991)

[LT] Lemaire, L.: Applications harmoniques de surfaces riemanniennes J. Diff. Geom. 13, 51-78 (1978)

[Ro] Robinson, D.C.: Uniqueness of the Kerr Black Hole. Phys. Rev. Lett. 34, 905-906 (1975)

[SU] Sacks, G., Uhlenbeck, K.: The existence of minimal immersions of two-spheres. Ann. Math. 113, 1-24 (1981)

[Sc] Schoen, R.: Analytic aspects of the harmonic maps problem. Publ. M.S.R.I. 2, 321-358 (1984)

[SU1] Schoen, R., Uhlenbeck, K.: A regularity theory for harmonic maps. J. Diff. Geom. 17, 307-335 (1982)

[SU2] Schoen, R.: Boundary regularity and the Dirichlet problem for harmonic maps. J. Diff. Geom. 18, 253-268 (1983)

[SY] Schoen, R., Yau, S.-T.: Existence of incompressible minimal surfaces and the topology of three dimensional manifolds with non-negative scalar curvature. Ann. Math. 110, 127-142 (1979)

[We1] Weinstein, G.: On rotating black holes in equilibrium in general relativity. Ph.D. thesis, at Courant Institute, New York University (1989)

[We2] Weinstein, G.: On rotating black holes in equilibrium in general relativity. Comm. Pure Appl. Math. 43, 903-948 (1990) 